\title{
ASYMPTOTIC EXPANSIONS OF EIGENVALUES BY BOTH THE CROUZEIX-RAVIART AND ENRICHED CROUZEIX-RAVIART ELEMENTS
}

\author{
JUN HU AND LIMIN MA
}

\begin{abstract}
Aвstract. Asymptotic expansions are derived for eigenvalues produced by both the CrouzeixRaviart element and the enriched Crouzeix-Raviart element. The expansions are optimal in the sense that extrapolation eigenvalues based on them admit a fourth order convergence provided that exact eigenfunctions are smooth enough. The major challenge in establishing the expansions comes from the fact that the canonical interpolation of both nonconforming elements lacks a crucial superclose property, and the nonconformity of both elements. The main idea is to employ the relation between the lowest-order mixed Raviart-Thomas element and the two nonconforming elements, and consequently make use of the superclose property of the canonical interpolation of the lowest-order mixed Raviart-Thomas element. To overcome the difficulty caused by the nonconformity, the commuting property of the canonical interpolation operators of both nonconforming elements is further used, which turns the consistency error problem into an interpolation error problem. Then, a series of new results are obtained to show the final expansions.
\end{abstract}

Keywords. eigenvalue problem, Crouzeix-Raviart element, enriched Crouzeix-Raviart element, asymptotic expansion,

AMS subject classifications. $65 \mathrm{~N} 30$.

\section{INTRODUCTION}

Asymptotic expansions of approximate solutions guarantee the efficiency of extrapolation methods. The classical analysis of asymptotic expansions is usually carried out by using the superclose property of the canonical interpolation of the element under consideration, see for instance [1, 3, 5, 13, 16-24] and the references therein. For the Crouzeix-Raviart (CR for short hereinafter) element, the extrapolation methods in [15] were examined to improve the accuracy of discrete eigenvalues from second order to fourth order numerically. But no asymptotic expansions were analyzed there to justify the experimental results. One major difficulty comes from the fact that the canonical interpolation of the CR element does not admit such a superclose property.

In this paper, asymptotic expansions of eigenvalues on uniform triangulations are explored for both the CR element and the enriched Crouzeix-Raviart (ECR for short hereinafter) element for the first time. Errors of eigenvalues by nonconforming elements admit

The authors were supported by NSFC projects 11625101 and 11421101. 
the following identity in [9]

$$
\lambda-\lambda_{h}=\left\|\nabla_{h}\left(u-u_{h}\right)\right\|_{0, \Omega}^{2}+2 a_{h}\left(u, u_{h}\right)-2 \lambda_{h}\left(u, u_{h}\right)-\lambda_{h}\left\|u-u_{h}\right\|_{0, \Omega}^{2}
$$

with approximate eigenpairs $\left(\lambda_{h}, u_{h}\right)$ defined in (2.4) below. Compared to conforming elements, there exist two major difficulties. The canonical interpolation of the nonconforming elements does not admit a superclose property. This leads to the difficulty in expanding the first term $\left\|\nabla_{h}\left(u-u_{h}\right)\right\|_{0, \Omega}^{2}$ with high accuracy. The nonconformity causes the other difficulty in expanding the consistency error term $a_{h}\left(u, u_{h}\right)-\lambda_{h}\left(u, u_{h}\right)$ for nonconforming elements.

One major idea to overcome the first difficulty is to employ the relation between the lowest-order mixed Raviart-Thomas element (RT for short hereinafter) and both the CR element and the ECR element, and to exploit the superconvergence result [10] for the RT element. This superconvergence property of the mixed element remedies the lack of the superclose property of the canonical interpolation of both nonconforming elements. To overcome the second difficulty, the main idea is to make use of the commuting property of the canonical interpolation operator of both nonconforming elements. This commuting property turns the consistency error term into an interpolation error term. Take the CR element as an example, it follows from the aforementioned superconvergence of the RT element, the commuting property of the CR element and the special relation between the CR element and the RT element [25] that

$$
\lambda-\lambda_{\mathrm{CR}}=\left\|\left(I-\Pi_{\mathrm{RT}}\right) \nabla u\right\|_{0, \Omega}^{2}+\frac{\lambda^{2} H^{2}}{144}+I_{\mathrm{CR}}+I_{\mathrm{RT}}+I_{\mathrm{CR}}^{1}+I_{\mathrm{CR}}^{2}+O\left(h^{4}|\ln h \| u|_{\frac{7}{2}, \Omega}^{2}\right),
$$

with $H$ defined in (2.2), the terms $I_{\mathrm{CR}}, I_{\mathrm{RT}}, I_{\mathrm{CR}}^{1}$ and $I_{\mathrm{CR}}^{2}$ defined in (3.9). Optimal expansions of eigenvalues require fourth-order accurate expansions of $\left\|\left(I-\Pi_{R T}\right) \nabla u\right\|_{0, \Omega}^{2}$ and $I_{C R}$ and also an optimal analysis of the other terms in (1.1).

There are three key terms for the expansions. The first one is $\left\|\left(I-\Pi_{R T}\right) \nabla u\right\|_{0, \Omega}^{2}$, whose optimal expansion needs to introduce an operator with some commuting property, and a refined analysis of the associated interpolation error. The second one is $I_{\mathrm{CR}}$ which contains two terms, one is essentially a consistency error and only admits a third order convergence which can not be improved. Hence, the direct use of the Cauchy-Schwarz inequality and the Taylor expansions of interpolation errors only leads to a suboptimal expansion. The idea is to decompose the first term of $I_{C R}$ into two terms: one cancels this consistency error, and the other term has an asymptotic expansion. One key result for the analysis is a crucial superconvergence of the inner product of the errors of the canonical interpolation of the CR element and the piecewise constant $L^{2}$ projection. The third term is $I_{\mathrm{CR}}^{1}$. For it, a direct combination of Cauchy-Schwarz inequality and the superclose property of the CR element only yields a suboptimal estimate. The idea here is to make use of the relation between the CR element and the RT element and decompose it into three terms: a vanishing term, a fourth order term and a remaining term. By using the commuting property of the canonical interpolation operator of the CR element, the discrete eigenvalue problem and an auxiliary discrete source problem, and fully exploring the properties of the piecewise constant $L^{2}$ projection operator and the uniformity of the mesh, this remaining term can be 
in some sense transferred to a consistency error. A key result is the superconvergence of the inner product of the errors of the piecewise constant $L^{2}$ projections of two CR element functions.

The remaining paper is organized as follows. Section 2 presents second order elliptic eigenvalue problems and some notations. Section 3 explores optimal asymptotic expansions of approximate eigenvalues of the CR element and analyzes the optimal convergence rate of eigenvalues by extrapolation methods. Section 4 deals with eigenvalues of the ECR element in a similar way to that of the CR element in Section 3. Section 5 presents some numerical tests.

\section{Notations and Preliminaries}

2.1. Notations. Given a nonnegative integer $k$ and a bounded domain $\Omega \subset \mathbb{R}^{2}$ with boundary $\partial \Omega$, let $W^{k, \infty}(\Omega, \mathbb{R}), H^{k}(\Omega, \mathbb{R}),\|\cdot\|_{k, \Omega}$ and $|\cdot|_{k, \Omega}$ denote the usual Sobolev spaces, norm, and semi-norm, respectively. And $H_{0}^{1}(\Omega, \mathbb{R})=\left\{u \in H^{1}(\Omega, \mathbb{R}):\left.u\right|_{\partial \Omega}=0\right\}$. Denote the standard $L^{2}(\Omega, \mathbb{R})$ inner product and $L^{2}(K, \mathbb{R})$ inner product by $(\cdot, \cdot)$ and $(\cdot, \cdot)_{0, K}$, respectively.

Suppose that $\Omega \subset \mathbb{R}^{2}$ is a bounded polygonal domain covered exactly by a shape-regular partition $\mathcal{T}_{h}$ into simplices. Let $|K|$ denote the area of element $K$ and $|e|$ the length of edge $e$. Let $h_{K}$ denote the diameter of element $K \in \mathcal{T}_{h}$ and $h=\max _{K \in \mathcal{T}_{h}} h_{K}$. Denote the set of all interior edges and boundary edges of $\mathcal{T}_{h}$ by $\mathcal{E}_{h}^{i}$ and $\mathcal{E}_{h^{\prime}}^{b}$, respectively, and $\mathcal{E}_{h}=\mathcal{E}_{h}^{i} \cup \mathcal{E}_{h}^{b}$. For any interior edge $e=K_{e}^{1} \cap K_{e}^{2}$, denote the element with larger global label by $K_{e}^{1}$ and the one with smaller global label by $K_{e}^{2}$. Denote the corresponding unit normal vector which points from $K_{e}^{1}$ to $K_{e}^{2}$ by $\mathbf{n}_{e}$. Let [·] be the jump of piecewise functions over edge $e$, namely

$$
\left.[v]\right|_{e}:=\left.v\right|_{K_{e}^{1}}-\left.v\right|_{K_{e}^{2}}
$$

for any piecewise function $v$. For $K \subset \mathbb{R}^{2}, r \in \mathbb{Z}^{+}$, let $P_{r}(K, \mathbb{R})$ be the space of all polynomials of degree not greater than $r$ on $K$. For $r \geq 1$, denote

$$
\nabla P_{r}\left(K, \mathbb{R}^{2}\right):=\left\{\nabla v: v \in P_{r}(K, \mathbb{R})\right\} .
$$

Denote the piecewise gradient operator and the piecewise hessian operator by $\nabla_{h}$ and $\nabla_{h^{\prime}}^{2}$ respectively.

Let element $K$ have vertices $\mathbf{p}_{i}=\left(p_{i 1}, p_{i 2}\right), 1 \leq i \leq 3$ oriented counterclockwise, and corresponding barycentric coordinates $\left\{\psi_{i}\right\}_{i=1}^{3}$. Let $\left\{e_{i}\right\}_{i=1}^{3}$ denote the edges of element $K,\left\{d_{i}\right\}_{i=1}^{3}$ the perpendicular heights, $\left\{\theta_{i}\right\}_{i=1}^{3}$ the internal angles, $\left\{\mathbf{m}_{i}\right\}_{i=1}^{3}$ the midpoint of edge $\left\{e_{i}\right\}_{i=1}^{3}$, and $\left\{\mathbf{n}_{i}\right\}_{i=1}^{3}$ the unit outward normal vectors, $\left\{\mathbf{t}_{i}\right\}_{i=1}^{3}$ the unit tangent vectors with counterclockwise orientation (see Figure 1). There holds the following relationships $d_{i}\left|e_{i}\right|=2|K|$ and

$$
\nabla \psi_{i}=-\frac{\mathbf{n}_{i}}{d_{i}}
$$

among the quantities [12]. Denote

$$
H_{K}^{2}=\sum_{i=1}^{3}\left|e_{i}\right|^{2} \quad\left(\text { on uniform meshes, } H_{K} \text { will be denoted by } H\right. \text { ) }
$$


and the centroid of element $K$ by $\mathbf{M}_{K}=\left(M_{1}, M_{2}\right)$. Denote the second order derivatives $\frac{\partial^{2} u}{\partial x_{i} \partial x_{j}}$ by $\partial_{x_{i} x_{j}} u, 1 \leq i, j \leq 2$. For ease of presentation, the symbol $A \lesssim B$ will be used to

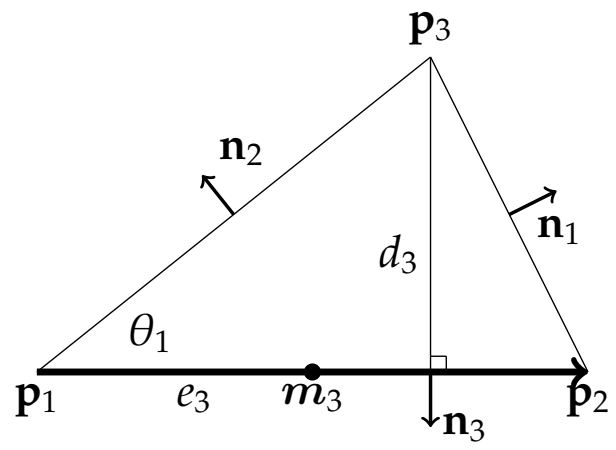

Figure 1. Paramters associated with a triangle $K$.

denote that $A \leq C B$, where $C$ is a positive constant.

2.2. Nonconforming elements for eigenvalue problems. Consider a model eigenvalue problem of finding : $(\lambda, u) \in \mathbb{R} \times V$ such that $\|u\|_{0, \Omega}=1$ and

$$
a(u, v)=\lambda(u, v) \quad \text { for any } v \in V,
$$

with $V:=H_{0}^{1}(\Omega, \mathbb{R})$. The bilinear form $a(w, v):=\int_{\Omega} \nabla w \cdot \nabla v d x$ is symmetric, bounded, and coercive, namely for any $w, v \in V$,

$$
a(w, v)=a(v, w), \quad|a(w, v)| \lesssim\|w\|_{1, \Omega}\|v\|_{1, \Omega}, \quad\|v\|_{1, \Omega}^{2} \lesssim a(v, v) .
$$

The eigenvalue problem (2.3) has a sequence of eigenvalues

$$
0<\lambda^{1} \leq \lambda^{2} \leq \lambda^{3} \leq \ldots \nearrow+\infty,
$$

and the corresponding eigenfunctions $u^{1}, u^{2}, u^{3}, \ldots$, with

$$
\left(u^{i}, u^{j}\right)=\delta_{i j} \text { with } \delta_{i j}=\left\{\begin{array}{ll}
0 & i \neq j \\
1 & i=j
\end{array} .\right.
$$

Let $V_{h}$ be a nonconforming finite element approximation of $V$ over $\mathcal{T}_{h}$. The corresponding finite element approximation of (2.3) is to find $\left(\lambda_{h}, u_{h}\right) \in \mathbb{R} \times V_{h}$ such that $\left\|u_{h}\right\|_{0, \Omega}=1$ and

$$
a_{h}\left(u_{h}, v_{h}\right)=\lambda_{h}\left(u_{h}, v_{h}\right) \quad \text { for any } v_{h} \in V_{h},
$$

with the discrete bilinear form $a_{h}\left(w_{h}, v_{h}\right):=\sum_{K \in \mathcal{T}_{h}} \int_{K} \nabla_{h} w_{h} \cdot \nabla_{h} v_{h} d x$.

Consider the following two nonconforming elements: the CR element and the ECR element. 
- The CR element space over $\mathcal{T}_{h}$ is defined in [4] by

$$
\begin{aligned}
V_{\mathrm{CR}}:= & \left\{v \in L^{2}(\Omega, \mathbb{R})|v|_{K} \in P_{1}(K, \mathbb{R}) \text { for any } K \in \mathcal{T}_{h}, \int_{e}[v] d s=0 \text { for any } e \in \mathcal{E}_{h}^{i},\right. \\
& \left.\int_{e} v d s=0 \text { for any } e \in \mathcal{E}_{h}^{b}\right\} .
\end{aligned}
$$

The corresponding canonical interpolation operator $\Pi_{\mathrm{CR}}: V \rightarrow V_{\mathrm{CR}}$ is defined as follows:

$$
\int_{e} \Pi_{\mathrm{CR}} v d s=\int_{e} v d s \quad \text { for any } e \in \mathcal{E}_{h}, v \in V .
$$

Denote the approximate eigenpair of (2.4) with $V_{h}=V_{\mathrm{CR}}$ by $\left(\lambda_{\mathrm{CR}}, u_{\mathrm{CR}}\right)$ with $\left\|u_{\mathrm{CR}}\right\|_{0, \Omega}=1$.

- The ECR element space over $\mathcal{T}_{h}$ is defined in [8] by

$$
\begin{aligned}
V_{\mathrm{ECR}}:= & \left\{v \in L^{2}(\Omega, \mathbb{R})|v|_{K} \in \operatorname{ECR}(\mathrm{K}, \mathbb{R}) \text { for any } \mathrm{K} \in \mathcal{T}_{\mathrm{h}}, \int_{\mathrm{e}}[\mathrm{v}] \mathrm{ds}=0 \text { for any e } \in \mathcal{E}_{\mathrm{h}}^{\mathrm{i}},\right. \\
& \left.\int_{e} v d s=0 \text { for any } e \in \mathcal{E}_{h}^{b}\right\} .
\end{aligned}
$$

with $\operatorname{ECR}(K, \mathbb{R}):=\mathrm{P}_{1}(\mathrm{~K}, \mathbb{R})+\operatorname{span}\left\{\mathrm{x}_{1}^{2}+\mathrm{x}_{2}^{2}\right\}$. The corresponding canonical interpolation operator $\Pi_{\mathrm{ECR}}: V \rightarrow V_{\mathrm{ECR}}$ is defined by

$$
\int_{e} \Pi_{\mathrm{ECR}} v d s=\int_{e} v d s, \quad \int_{K} \Pi_{\mathrm{ECR}} v d x=\int_{K} v d x \quad \text { for any } e \in \mathcal{E}_{h}, K \in \mathcal{T}_{h} .
$$

Denote the approximate eigenpair of (2.4) $V_{h}=V_{\mathrm{ECR}}$ by $\left(\lambda_{\mathrm{ECR}}, u_{\mathrm{ECR}}\right)$ and $\left\|u_{\mathrm{ECR}}\right\|_{0, \Omega}=1$.

Assumption 2.1. The domain $\Omega$ is convex or eigenfunction $u$ is smooth.

Assumption 2.1 guarantees that eigenfunctions belong to $H^{2}(\Omega, \mathbb{R})$. It follows from the theory of nonconforming eigenvalue approximations, see for instance, [8, 28] and the references therein, that

$$
\begin{gathered}
\left|\lambda-\lambda_{\mathrm{CR}}\right|+\left\|u-u_{\mathrm{CR}}\right\|_{0, \Omega}+\left\|u-\Pi_{\mathrm{CR}} u\right\|_{0, \Omega}+h\left\|\nabla_{h}\left(u-u_{\mathrm{CR}}\right)\right\|_{0, \Omega} \lesssim h^{2}\|u\|_{2, \Omega}, \\
\left|\lambda-\lambda_{\mathrm{ECR}}\right|+\left\|u-u_{\mathrm{ECR}}\right\|_{0, \Omega}+\left\|u-\Pi_{\mathrm{ECR}} u\right\|_{0, \Omega}+h\left\|\nabla_{h}\left(u-u_{\mathrm{ECR}}\right)\right\|_{0, \Omega} \lesssim h^{2}\|u\|_{2, \Omega} .
\end{gathered}
$$

For the CR element and the ECR element, there holds the following commuting property for their canonical interpolations

$$
\begin{array}{cc}
\int_{K} \nabla\left(w-\Pi_{\mathrm{CR}} w\right) \cdot \nabla v_{h} d x=0 & \text { for any } w \in V, v_{h} \in V_{\mathrm{CR}} \\
\int_{K} \nabla\left(w-\Pi_{\mathrm{ECR}} w\right) \cdot \nabla v_{h} d x=0 & \text { for any } w \in V, v_{h} \in V_{\mathrm{ECR}}
\end{array}
$$

see [4, 8] for more details.

For the CR element, there exists the following identity for the error of the approximate eigenvalues [9]

$$
\lambda-\lambda_{\mathrm{CR}}=\left\|\nabla_{h}\left(u-u_{\mathrm{CR}}\right)\right\|_{0, \Omega}^{2}+2 a_{h}\left(u, u_{\mathrm{CR}}\right)-2 \lambda_{\mathrm{CR}}\left(u, u_{\mathrm{CR}}\right)-\lambda_{\mathrm{CR}}\left\|u-u_{\mathrm{CR}}\right\|_{0, \Omega}^{2} .
$$


Jun $\mathrm{Hu}$ and Limin Ma

It is difficult to establish an asymptotic expansion for the consistency error term $a_{h}\left(u, u_{\mathrm{CR}}\right)-$ $\lambda_{\mathrm{CR}}\left(u, u_{\mathrm{CR}}\right)$ directly. The main idea herein is to employ the canonical interpolation operator $\Pi_{C R}$ of the CR element and the crucial commuting property (2.9). In this way, the consistency error term can be expressed in terms of the interpolation error, namely

$$
a_{h}\left(u, u_{\mathrm{CR}}\right)-\lambda_{\mathrm{CR}}\left(u, u_{\mathrm{CR}}\right)=-\lambda_{\mathrm{CR}}\left(u-\Pi_{\mathrm{CR}} u, u_{\mathrm{CR}}\right) .
$$

As a result, the identity (2.10) becomes

$$
\lambda-\lambda_{\mathrm{CR}}=\left\|\nabla_{h}\left(u-u_{\mathrm{CR}}\right)\right\|_{0, \Omega}^{2}-2 \lambda_{\mathrm{CR}}\left(u-\Pi_{\mathrm{CR}} u, u_{\mathrm{CR}}\right)-\lambda_{\mathrm{CR}}\left\|u-u_{\mathrm{CR}}\right\|_{0, \Omega}^{2} .
$$

The asymptotic expansions of eigenvalues of the CR element in this paper are based on this crucial identity (2.11). Since the ECR element also admits a commuting property, a similar identity to (2.11) holds for approximate eigenpairs $\left(\lambda_{\mathrm{ECR}}, u_{\mathrm{ECR}}\right)$, and leads to the asymptotic expansions of eigenvalues of the ECR element.

2.3. Raviart-Thomas element for source problems. The shape function space of the lowest order RT element [29] is as follows

$$
\mathrm{RT}\left(\mathrm{K}, \mathbb{R}^{2}\right):=\mathrm{P}_{0}\left(\mathrm{~K}, \mathbb{R}^{2}\right)+\mathbf{x} \mathrm{P}_{0}(\mathrm{~K}, \mathbb{R}) \quad \text { for any } \mathrm{K} \in \mathcal{T}_{\mathrm{h}} .
$$

The corresponding finite element space reads

$$
\operatorname{RT}\left(\mathcal{T}_{h}\right):=\left\{\tau \in H\left(\operatorname{div}, \Omega, \mathbb{R}^{2}\right):\left.\tau\right|_{K} \in \mathrm{RT}\left(\mathrm{K}, \mathbb{R}^{2}\right) \text { for any } \mathrm{K} \in \mathcal{T}_{\mathrm{h}}\right\} .
$$

To get a stable pair of space, the piecewise constant space is used to approximate the displacement, namely,

$$
U_{\mathrm{RT}}:=\left\{v \in L^{2}(\Omega, \mathbb{R}):\left.v\right|_{K} \in P_{0}(K, \mathbb{R}) \text { for any } K \in \mathcal{T}_{h}\right\} .
$$

The Fortin interpolation operator $\prod_{\mathrm{RT}}: H\left(\operatorname{div}, \Omega, \mathbb{R}^{2}\right) \rightarrow \mathrm{RT}\left(\mathcal{T}_{\mathrm{h}}\right)$, which is widely used in error analysis, see for instance [6, 7] and the references therein, is defined by

$$
\int_{e}\left(\Pi_{\mathrm{RT}} \tau-\tau\right)^{T} \mathbf{n}_{e} d s=0 \quad \text { for any } e \in \mathcal{E}_{h}, \tau \in H\left(\operatorname{div}, \Omega, \mathbb{R}^{2}\right) .
$$

For the analysis, introduce the following discrete source problem which seeks $\left(\sigma_{\mathrm{RT}}^{\lambda u}, u_{\mathrm{RT}}^{\lambda u}\right) \in$ $\mathrm{RT}\left(\mathcal{T}_{h}\right) \times U_{\mathrm{RT}}$ such that

$$
\begin{aligned}
\left(\sigma_{\mathrm{RT}}^{\lambda u}, \tau_{h}\right)+\left(u_{\mathrm{RT}}^{\lambda u}, \operatorname{div} \tau_{h}\right) & =0 & & \text { for any } \tau_{h} \in \mathrm{RT}\left(\mathcal{T}_{h}\right), \\
\left(\operatorname{div} \sigma_{\mathrm{RT}}^{\lambda u}, v_{h}\right) & =-\lambda\left(u, v_{h}\right) & & \text { for any } v_{h} \in U_{\mathrm{RT}} .
\end{aligned}
$$

Note that $\sigma_{\mathrm{RT}}^{\lambda u}$ is the RT element solution of $\sigma^{\lambda \mathrm{u}}=\nabla u$. It follows from the theory of mixed finite element methods [6] that

$$
\left\|u-u_{\mathrm{RT}}^{\lambda u}\right\|_{0, \Omega}+\left\|\nabla u-\sigma_{\mathrm{RT}}^{\lambda u}\right\|_{0, \Omega}+\left\|\operatorname{div}\left(\sigma^{\lambda \mathrm{u}}-\sigma_{\mathrm{RT}}^{\lambda \mathrm{u}}\right)\right\|_{0, \Omega} \lesssim \mathrm{h}^{\mathrm{s}}\|\mathrm{u}\|_{1+\mathrm{s}, \Omega},
$$

provided that $u \in H^{1+s}(\Omega, \mathbb{R}) \cap H_{0}^{1}(\Omega, \mathbb{R}), 0<s \leq 1$.

In this paper, the family of triangulations is assumed to be uniform:

Assumption 2.2. The triangulation $\mathcal{T}_{h}$ is uniform. This is, any two adjacent triangles form a parallelogram. 
In this paper, only uniform meshes in this sense will be used. For this case, the mesh dependent constant $H_{K}$ from (2.2) will be denoted by $H$.

According to [2, 10, 14], the RT element admits an important superconvergence property on uniform triangulations.

Lemma 2.1. Suppose that $\left(\sigma_{\mathrm{RT}}^{\lambda u}, u_{\mathrm{RT}}^{\lambda u}\right)$ is the solution of problem (2.13) and $u \in H^{\frac{7}{2}}(\Omega, \mathbb{R}) \cap$ $H_{0}^{1}(\Omega, \mathbb{R})$. Under the Assumption 2.2 .

$$
\left\|\sigma_{\mathrm{RT}}^{\lambda u}-\Pi_{\mathrm{RT}} \nabla u\right\|_{0, \Omega} \lesssim h^{2}\left(|u|_{\frac{7}{2}, \Omega}+\kappa|\ln h|^{1 / 2}|u|_{2, \infty, \Omega}\right) .
$$

2.4. Relation between the RT element and nonconforming elements. Define the $L^{2}$ projection operators $\Pi_{K}^{0}: L^{2}(K, \mathbb{R}) \rightarrow P_{0}(K, \mathbb{R})$ and $\Pi_{h}^{0}: L^{2}(\Omega, \mathbb{R}) \rightarrow U_{\mathrm{RT}}$ by

$$
\Pi_{K}^{0} w=\frac{1}{|K|} \int_{K} w d x \quad \text { and }\left.\quad \Pi_{h}^{0} w\right|_{K}=\Pi_{K}^{0} u,
$$

respectively. It holds that for any $u \in H^{1}(K, \mathbb{R})$,

$$
\left\|u-\Pi_{K}^{0} u\right\|_{0, K} \lesssim h|u|_{1, K} .
$$

Consider two discrete source problems: one seeks $u_{\mathrm{CR}}^{\lambda \Pi_{h}^{0} u} \in V_{\mathrm{CR}}$ such that

$$
\left(\nabla_{h} u_{\mathrm{CR}}^{\lambda \Pi_{h}^{0} u}, \nabla_{h} v_{h}\right)=\lambda\left(\Pi_{h}^{0} u, v_{h}\right) \quad \text { for any } v_{h} \in V_{\mathrm{CR}}
$$

and the other one seeks $u_{\mathrm{ECR}}^{\lambda \Pi_{h}^{0} u} \in V_{\mathrm{ECR}}$ such that

$$
\left(\nabla_{h} u_{\mathrm{ECR}}^{\lambda \Pi_{h}^{0} u}, \nabla_{h} v_{h}\right)=\left(\lambda \Pi_{h}^{0} u, v_{h}\right) \quad \text { for any } v_{h} \in V_{\mathrm{ECR}} .
$$

By the definition of the $L^{2}$ projection operator $\Pi_{h}^{0}$ and the discrete space $U_{\mathrm{RT}}$, it holds that

$$
\left(\lambda u, v_{h}\right)=\left(\lambda \Pi_{h}^{0} u, v_{h}\right) \text {, for any } v_{h} \in U_{\mathrm{RT}} .
$$

This implies that the discrete source problem (2.13) can be rewritten as: finding $\left(\sigma_{\mathrm{RT}}^{\lambda u}, u_{\mathrm{RT}}^{\lambda u}\right) \in$ $\mathrm{RT}\left(\mathcal{T}_{h}\right) \times U_{\mathrm{RT}}$ such that

$$
\begin{aligned}
\left(\sigma_{\mathrm{RT}}^{\lambda u}, \tau_{h}\right)+\left(u_{\mathrm{RT}}^{\lambda u}, \operatorname{div} \tau_{h}\right) & =0 & & \text { for any } \tau_{h} \in \mathrm{RT}\left(\mathcal{T}_{h}\right), \\
\left(\operatorname{div} \sigma_{\mathrm{RT}}^{\lambda u}, v_{h}\right) & =-\lambda\left(\Pi_{h}^{0} u, v_{h}\right) & & \text { for any } v_{h} \in U_{\mathrm{RT}} .
\end{aligned}
$$

The following Lemma states the special relations between the RT solution of Problem (2.13) and the CR solution of Problem (2.18), and the ECR solution of Problem (2.19), respectively, see more details in [25] and [11].

Lemma 2.2. Let $\left(\sigma_{\mathrm{RT}}^{\lambda u}, u_{\mathrm{RT}}^{\lambda u}\right), u_{\mathrm{CR}}^{\lambda \Pi_{h}^{0} u}$ and $u_{\mathrm{ECR}}^{\lambda \Pi_{h}^{0} u}$ be the solutions of (2.13), (2.18) and (2.19), respectively. It holds that

$$
\begin{gathered}
\left.\sigma_{\mathrm{RT}}^{\lambda u}\right|_{K}=\left.\nabla_{h} u_{\mathrm{CR}}^{\lambda \Pi_{h}^{0} u}\right|_{K}-\frac{\lambda \Pi_{K}^{0} u}{2}\left(\mathbf{x}-\mathbf{M}_{K}\right), \quad \mathbf{x} \in K, \\
\sigma_{\mathrm{RT}}^{\lambda u}=\nabla_{h} u_{\mathrm{ECR}}^{\lambda \Pi_{h}^{0} u},
\end{gathered}
$$

for any $K \in \mathcal{T}_{h}$ with $\mathbf{M}_{K}$ the centroid of $K$. 


\section{Asymptotic eXPANSions of eigenvalues of the CR Element}

In this section, asymptotic expansions of eigenvalues are established for the CR element, and then employed to prove an optimal convergence of eigenvalues by extrapolation methods.

3.1. Error expansions for eigenvalues. Let $u_{\mathrm{CR}}^{\lambda u} \in V_{\mathrm{CR}}$ be the solution of the following source problem

$$
\left(\nabla_{h} u_{\mathrm{CR}}^{\lambda u}, \nabla_{h} v_{h}\right)=\lambda\left(u, v_{h}\right) \quad \text { for any } v_{h} \in V_{\mathrm{CR}}
$$

where $(\lambda, u)$ is the solution of the eigenvalue problem (2.3). The following lemma presents a superclose property of the discrete eigenfunction $u_{\mathrm{CR}}$ with respect to the CR solution $u_{\mathrm{CR}}^{\lambda \Pi_{h}^{0} u}$ of the discrete source problem (2.18).

Lemma 3.1. Suppose that $(\lambda, u)$ is the eigenpair of (2.3), $\left(\lambda_{\mathrm{CR}}, u_{\mathrm{CR}}\right)$ is the corresponding eigenpair of (2.4) by the CR element and $u_{\mathrm{CR}}^{\lambda \Pi_{h}^{0} u}$ is the solution of (2.18). Under the Assumption 2.1.

$$
\left\|\nabla_{h} u_{\mathrm{CR}}-\nabla_{h} u_{\mathrm{CR}}^{\lambda \Pi_{h}^{0} u}\right\|_{0, \Omega} \lesssim h^{2}|u|_{2, \Omega} .
$$

Proof. It follows from the triangle inequality that

$$
\left\|\nabla_{h} u_{\mathrm{CR}}-\nabla_{h} u_{\mathrm{CR}}^{\lambda \Pi_{h}^{0} u}\right\|_{0, \Omega} \leq\left\|\nabla_{h} u_{\mathrm{CR}}-\nabla_{h} u_{\mathrm{CR}}^{\lambda u}\right\|_{0, \Omega}+\left\|\nabla_{h} u_{\mathrm{CR}}^{\lambda u}-\nabla_{h} u_{\mathrm{CR}}^{\lambda \Pi_{h}^{0} u}\right\|_{0, \Omega} \text {. }
$$

To bound the first term on the right-hand side of (3.3), let $v_{h}=u_{\mathrm{CR}}-u_{\mathrm{CR}}^{\lambda u}$ in Problem (2.4) and Problem (3.1). This yields

$$
\left\|\nabla_{h} u_{\mathrm{CR}}-\nabla_{h} u_{\mathrm{CR}}^{\lambda u}\right\|_{0, \Omega}^{2}=\left(\lambda_{\mathrm{CR}} u_{\mathrm{CR}}-\lambda u, u_{\mathrm{CR}}-u_{\mathrm{CR}}^{\lambda u}\right) \text {. }
$$

By the error estimate (2.7) for the discrete eigenpair $\left(\lambda_{\mathrm{CR}}, u_{\mathrm{CR}}\right)$,

$$
\left\|\lambda_{\mathrm{CR}} u_{\mathrm{CR}}-\lambda u\right\|_{0, \Omega} \leq\left|\lambda_{\mathrm{CR}}\right|\left\|u_{\mathrm{CR}}-u\right\|_{0, \Omega}+\left|\lambda_{\mathrm{CR}}-\lambda\right| \lesssim h^{2}|u|_{2, \Omega} \text {. }
$$

It follows from the triangle inequality, the error estimate (2.7), and the fact that $u_{\mathrm{CR}}^{\lambda u}$ is the CR element approximation of the solution $u$ of the source problem $-\Delta w=\lambda u$ with $(\lambda, u)$ the eigenpair of Problem (2.3), that

$$
\left\|u_{\mathrm{CR}}-u_{\mathrm{CR}}^{\lambda u}\right\|_{0, \Omega} \leq\left\|u_{\mathrm{CR}}-u\right\|_{0, \Omega}+\left\|u-u_{\mathrm{CR}}^{\lambda u}\right\|_{0, \Omega} \lesssim h^{2}|u|_{2, \Omega} .
$$

A substitution of (3.5) and (3.6) to (3.4) leads to

$$
\left\|\nabla_{h} u_{\mathrm{CR}}-\nabla_{h} u_{\mathrm{CR}}^{\lambda u}\right\|_{0, \Omega} \lesssim h^{2}|u|_{2, \Omega} \text {. }
$$

To bound the second term on the right-hand side of (3.3), let $v_{h}=u_{\mathrm{CR}}-u_{\mathrm{CR}}^{\lambda u}$ in Problem (2.18) and Problem (3.1). This yields

$$
\left\|\nabla_{h} u_{\mathrm{CR}}^{\lambda u}-\nabla_{h} u_{\mathrm{CR}}^{\lambda \Pi_{h}^{0} u}\right\|_{0, \Omega}^{2}=\lambda\left(u-\Pi_{h}^{0} u, u_{\mathrm{CR}}^{\lambda u}-u_{\mathrm{CR}}^{\lambda \Pi_{h}^{0} u}\right)=\lambda\left(u-\Pi_{h}^{0} u,\left(I-\Pi_{h}^{0}\right)\left(u_{\mathrm{CR}}^{\lambda u}-u_{\mathrm{CR}}^{\lambda \Pi_{h}^{0} u}\right)\right) \text {. }
$$

This, together with the error estimate (2.17) of the piecewise constant $L^{2}$-projection $\Pi_{h}^{0} u$ and a Poincare inequality for the term $\left(I-\Pi_{h}^{0}\right)\left(u_{\mathrm{CR}}^{\lambda u}-u_{\mathrm{CR}}^{\lambda \Pi_{h}^{0} u}\right)$, leads to

$$
\left\|\nabla_{h} u_{\mathrm{CR}}^{\lambda u}-\nabla_{h} u_{\mathrm{CR}}^{\lambda \Pi_{h}^{0} u}\right\|_{0, \Omega}^{2} \lesssim \lambda h^{2}|u|_{1, \Omega}\left\|\nabla_{h} u_{\mathrm{CR}}^{\lambda u}-\nabla_{h} u_{\mathrm{CR}}^{\lambda \Pi_{h}^{0} u}\right\|_{0, \Omega},
$$


consequently,

$$
\left\|\nabla_{h} u_{\mathrm{CR}}^{\lambda u}-\nabla_{h} u_{\mathrm{CR}}^{\lambda \Pi_{h}^{0} u}\right\|_{0, \Omega} \lesssim \lambda h^{2}|u|_{1, \Omega} .
$$

A substitution of (3.7) and (3.8) to (3.3) leads to

$$
\left\|\nabla_{h} u_{\mathrm{CR}}-\nabla_{h} u_{\mathrm{CR}}^{\lambda \Pi_{h}^{0} u}\right\|_{0, \Omega} \lesssim h^{2}|u|_{2, \Omega}
$$

and completes the proof.

For the CR element, the canonical interpolation does not admit the usual superclose property with respect to the finite element solution in the energy norm. The lack of this crucial superclose property makes it difficult to establish asymptotic expansions of eigenvalues by directly using the canonical interpolation of the nonconforming CR element.

To overcome such a difficulty, the key idea is to make use of the relation between the RT element and the CR element in Lemma 2.2 and the superconvergence property of the mixed RT element. To this end, introduce the following notations with

$$
\begin{aligned}
& I_{\mathrm{CR}}=2\left(\left(I-\Pi_{\mathrm{RT}}\right) \nabla u, \sigma_{\mathrm{RT}}^{\lambda u}-\nabla_{h} u_{\mathrm{CR}}^{\lambda \Pi_{h}^{0} u}\right)-2 \lambda\left(u-\Pi_{\mathrm{CR}} u, u\right), \\
& I_{\mathrm{RT}}=2\left(\nabla u-\Pi_{\mathrm{RT}} \nabla u, \Pi_{\mathrm{RT}} \nabla u-\sigma_{\mathrm{RT}}^{\lambda u}\right), \\
& I_{\mathrm{CR}}^{1}=2\left(\nabla u-\Pi_{\mathrm{RT}} \nabla u, \nabla_{h} u_{\mathrm{CR}}^{\lambda \Pi_{h}^{0} u}-\nabla_{h} u_{\mathrm{CR}}\right), \\
& I_{\mathrm{CR}}^{2}=2\left(\Pi_{\mathrm{RT}} \nabla u-\sigma_{\mathrm{RT}}^{\lambda u}, \sigma_{\mathrm{RT}}^{\lambda u}-\nabla_{h} u_{\mathrm{CR}}\right) .
\end{aligned}
$$

The asymptotic expansions of eigenvalues of the CR element are based on the identity in the following theorem.

Theorem 3.1. Suppose that $(\lambda, u)$ is the eigenpair of $(2.3)$ with $u \in H^{\frac{7}{2}}(\Omega, \mathbb{R}) \cap H_{0}^{1}(\Omega, \mathbb{R})$, and $\left(\lambda_{\mathrm{CR}}, u_{\mathrm{CR}}\right)$ is the discrete eigenpair of (2.4) by the CR element. Under Assumption 2.2.

$$
\lambda-\lambda_{\mathrm{CR}}=\left\|\left(I-\Pi_{\mathrm{RT}}\right) \nabla u\right\|_{0, \Omega}^{2}+\frac{\lambda^{2} H^{2}}{144}+I_{\mathrm{CR}}+I_{\mathrm{RT}}+I_{\mathrm{CR}}^{1}+I_{\mathrm{CR}}^{2}+O\left(h^{4}|\ln h \| u|_{\frac{7}{2}, \Omega}^{2}\right),
$$

with $H$ defined in (2.2), and $I_{\mathrm{CR}}, I_{\mathrm{RT}}, I_{\mathrm{CR}}^{1}$ and $I_{\mathrm{CR}}^{2}$ defined in (3.9).

Proof. Recall the expansion (2.11) of eigenvalues by the CR element as follows

$$
\lambda-\lambda_{\mathrm{CR}}=\left\|\nabla_{h}\left(u-u_{\mathrm{CR}}\right)\right\|_{0, \Omega}^{2}-2 \lambda_{\mathrm{CR}}\left(u-\Pi_{\mathrm{CR}} u, u_{\mathrm{CR}}\right)-\lambda_{\mathrm{CR}}\left\|u-u_{\mathrm{CR}}\right\|_{0, \Omega}^{2}
$$

The second term on the right-hand side can be decomposed into three terms as follows

$$
\begin{aligned}
\lambda_{\mathrm{CR}}\left(u-\Pi_{\mathrm{CR}} u, u_{\mathrm{CR}}\right)= & \lambda\left(u-\Pi_{\mathrm{CR}} u, u\right)+\left(\lambda_{\mathrm{CR}}-\lambda\right)\left(u-\Pi_{\mathrm{CR}} u, u\right) \\
& +\lambda_{\mathrm{CR}}\left(u-\Pi_{\mathrm{CR}} u, u_{\mathrm{CR}}-u\right) .
\end{aligned}
$$

By the error estimates (2.7) of the CR element,

$$
\begin{gathered}
\left|\left(\lambda_{\mathrm{CR}}-\lambda\right)\left(u-\Pi_{\mathrm{CR}} u, u\right)\right| \lesssim\left|\lambda_{\mathrm{CR}}-\lambda\right|\left\|u-\Pi_{\mathrm{CR}} u\right\|_{0, \Omega} \lesssim h^{4}|u|_{2, \Omega}^{2}, \\
\left|\left(u-\Pi_{\mathrm{CR}} u, u_{\mathrm{CR}}-u\right)\right| \lesssim|| u-\Pi_{\mathrm{CR}} u\left\|_{0, \Omega}\right\| u_{\mathrm{CR}}-u \|_{0, \Omega} \lesssim h^{4}|u|_{2, \Omega}^{2} .
\end{gathered}
$$


A substitution of these two estimates into (3.11) yields

$$
\lambda_{\mathrm{CR}}\left(u-\Pi_{\mathrm{CR}} u, u_{\mathrm{CR}}\right)=\lambda\left(u-\Pi_{\mathrm{CR}} u, u\right)+O\left(h^{4}|u|_{2, \Omega}^{2}\right) .
$$

Note that

$$
\nabla u-\nabla_{h} u_{\mathrm{CR}}=\left(\nabla u-\Pi_{\mathrm{RT}} \nabla u\right)+\left(\Pi_{\mathrm{RT}} \nabla u-\sigma_{\mathrm{RT}}^{\lambda u}\right)+\left(\sigma_{\mathrm{RT}}^{\lambda u}-\nabla_{h} u_{\mathrm{CR}}^{\lambda \Pi_{h}^{0} u}\right)+\left(\nabla_{h} u_{\mathrm{CR}}^{\lambda \Pi_{h}^{0} u}-\nabla_{h} u_{\mathrm{CR}}\right),
$$

with the RT solution $\sigma_{\mathrm{RT}}^{\lambda u}$ of the source problem (2.13) and the CR solution $u_{\mathrm{CR}}^{\lambda \Pi_{h}^{0} u}$ of the modified problem (2.18). A substitution of the above decomposition and (3.12) into (2.11) leads to

$$
\begin{aligned}
\lambda-\lambda_{\mathrm{CR}}= & \left\|\nabla u-\Pi_{\mathrm{RT}} \nabla u\right\|_{0, \Omega}^{2}+\left\|\Pi_{\mathrm{RT}} \nabla u-\sigma_{\mathrm{RT}}^{\lambda u}\right\|_{0, \Omega}^{2}+\left\|\sigma_{\mathrm{RT}}^{\lambda u}-\nabla_{h} u_{\mathrm{CR}}^{\lambda \Pi_{h}^{0} u}\right\|_{0, \Omega}^{2} \\
& +\left\|\nabla_{h} u_{\mathrm{CR}}^{\lambda \Pi_{h}^{0} u}-\nabla_{h} u_{\mathrm{CR}}\right\|_{0, \Omega}^{2}+2\left(\sigma_{\mathrm{RT}}^{\lambda u}-\nabla_{h} u_{\mathrm{CR}}^{\lambda \Pi_{h}^{0} u}, \nabla_{h} u_{\mathrm{CR}}^{\lambda \Pi_{h}^{0} u}-\nabla_{h} u_{\mathrm{CR}}\right) \\
& +I_{\mathrm{CR}}+I_{\mathrm{RT}}+I_{\mathrm{CR}}^{1}+I_{\mathrm{CR}}^{2}+O\left(h^{4}|u|_{2, \Omega}^{2}\right)
\end{aligned}
$$

with $I_{\mathrm{CR}}, I_{\mathrm{RT}}, I_{\mathrm{CR}}^{1}$ and $I_{\mathrm{CR}}^{2}$ defined in (3.9). By the definition of the $L^{2}$ projection operator $\Pi_{K}^{0}$ and the corresponding error estimate (2.17),

$$
\left\|\Pi_{K}^{0} u\right\|_{0, K}^{2}=\|u\|_{0, K}^{2}-\left\|u-\Pi_{K}^{0} u\right\|_{0, K}^{2} \quad \text { with } \quad\left\|u-\Pi_{K}^{0} u\right\|_{0, K} \lesssim h|u|_{1, K} .
$$

According to the relation (2.20) between the CR element and the RT element, namely $\left.2\left(\sigma_{\mathrm{RT}}^{\lambda u}-\nabla_{h} u_{\mathrm{CR}}^{\lambda \Pi_{h}^{0} u}\right)\right|_{K}=-\lambda \Pi_{K}^{0} u\left(\mathbf{x}-\mathbf{M}_{K}\right)$, this leads to

$$
\left\|\sigma_{\mathrm{RT}}^{\lambda u}-\nabla_{h} u_{\mathrm{CR}}^{\lambda \Pi_{h}^{0} u}\right\|_{0, \Omega}^{2}=\frac{\lambda^{2}}{144} \sum_{K \in \mathcal{T}_{h}} H^{2}\|u\|_{0, K}^{2}+O\left(h^{4}|u|_{1, \Omega}^{2}\right) .
$$

It follows from the facts $\int_{K} \mathbf{x}-\mathbf{M}_{K} d x=0$ and $\nabla_{h} u_{\mathrm{CR}}^{\lambda \Pi_{h}^{0} u}-\left.\nabla_{h} u_{\mathrm{CR}}\right|_{K} \in P_{0}\left(K, \mathbb{R}^{2}\right)$ that

$$
2\left(\sigma_{\mathrm{RT}}^{\lambda u}-\nabla_{h} u_{\mathrm{CR}}^{\lambda \Pi_{h}^{0} u}, \nabla_{h} u_{\mathrm{CR}}^{\lambda \Pi_{h}^{0} u}-\nabla_{h} u_{\mathrm{CR}}\right)=-\lambda\left(\Pi_{h}^{0} u\left(\mathbf{x}-\mathbf{M}_{K}\right), \nabla_{h} u_{\mathrm{CR}}^{\lambda \Pi_{h}^{0} u}-\nabla_{h} u_{\mathrm{CR}}\right)=0 .
$$

Recall the superconvergence (2.15) of the RT element in Lemma 2.1

$$
\left\|\Pi_{\mathrm{RT}} \nabla u-\sigma_{\mathrm{RT}}^{\lambda u}\right\|_{0, \Omega} \lesssim h^{2}\left(|u|_{\frac{7}{2}, \Omega}+\kappa|\ln h|^{1 / 2}|u|_{2, \infty, \Omega}\right),
$$

and the superclose property (3.2) of the CR element in Lemma 3.1

$$
\left\|\nabla_{h} u_{\mathrm{CR}}^{\lambda \Pi_{h}^{0} u}-\nabla_{h} u_{\mathrm{CR}}\right\|_{0, \Omega} \lesssim h^{2}|u|_{2, \Omega} .
$$

Note that $\|u\|_{0, \Omega}=1$. A substitution of (2.15) , (3.2), (3.14) and (3.15) into (3.13) leads to

$$
\lambda-\lambda_{\mathrm{CR}}=\left\|\left(I-\Pi_{\mathrm{RT}}\right) \nabla u\right\|_{0, \Omega}^{2}+\frac{\lambda^{2} H^{2}}{144}+I_{\mathrm{CR}}+I_{\mathrm{RT}}+I_{\mathrm{CR}}^{1}+I_{\mathrm{CR}}^{2}+O\left(h^{4}|\ln h \| u|_{\frac{7}{2}, \Omega}^{2}\right),
$$

which completes the proof. 
According to Theorem 3.1 , the asymptotic expansion of eigenvalues requires the analysis of the following five terms

$$
\left\|\left(I-\Pi_{\mathrm{RT}}\right) \nabla u\right\|_{0, \Omega}^{2}, I_{\mathrm{CR}}, I_{\mathrm{RT}}, I_{\mathrm{CR}}^{1}, I_{\mathrm{CR}}^{2} .
$$

The fourth-order accurate expansions of the first two terms are analyzed in Section 3.2 and 3.3. respectively. Optimal estimates of $I_{\mathrm{RT}}, I_{\mathrm{CR}}^{1}$ and $I_{\mathrm{CR}}^{2}$ are analyzed in Section 3.4, 3.5 and 3.6. respectively.

3.2. Taylor expansion of $\left\|\left(I-\Pi_{\mathrm{RT}}\right) \nabla u\right\|_{0, \Omega}^{2}$. Define two short-hand notations for the RT element

$$
\phi_{\mathrm{RT}}^{1}(\mathbf{x})=\left(x_{1}-M_{1},-x_{2}+M_{2}\right)^{T}, \quad \phi_{\mathrm{RT}}^{2}(\mathbf{x})=\left(x_{2}-M_{2}, x_{1}-M_{1}\right)^{T} .
$$

Note that for $i=1$ and 2,

$$
\operatorname{div} \phi_{\mathrm{RT}}^{\mathrm{i}}=0, \quad \nabla \mathrm{P}_{2}\left(\mathrm{~K}, \mathbb{R}^{2}\right)=\mathrm{RT}\left(\mathrm{K}, \mathbb{R}^{2}\right)+\operatorname{span}\left\{\phi_{\mathrm{RT}}^{1}, \phi_{\mathrm{RT}}^{2}\right\} .
$$

Define

$$
\gamma_{\mathrm{RT}}^{i j}:=\frac{1}{h^{2}|K|} \int_{K}\left(\left(I-\Pi_{\mathrm{RT}}\right) \phi_{\mathrm{RT}}^{i}\right)^{T}\left(I-\Pi_{\mathrm{RT}}\right) \phi_{\mathrm{RT}}^{j} d x .
$$

Lemma 3.2. Under Assumption 2.2, constants $\gamma_{\mathrm{RT}}^{i j}$ in (3.16) are independent of the mesh size $h$.

Proof. Notice that

$$
\Pi_{\mathrm{RT}} \phi_{\mathrm{RT}}^{i}=\sum_{j=1}^{3} a_{\mathrm{RT}}^{i j}\left(\mathbf{x}-\mathbf{p}_{j}\right) \quad \text { with } \quad a_{\mathrm{RT}}^{i j}=\frac{1}{2|K|} \int_{e_{j}}\left(\phi_{\mathrm{RT}}^{i}\right)^{T} \mathbf{n}_{j} d s .
$$

Since $\phi_{\mathrm{RT}}^{i} \in P_{1}\left(K, \mathbb{R}^{2}\right)$,

$$
a_{\mathrm{RT}}^{i j}=\frac{\left|e_{j}\right|}{2|K|}\left(\phi_{\mathrm{RT}}^{i}\left(\mathbf{m}_{j}\right)\right)^{T} \mathbf{n}_{j}
$$

with $\mathbf{m}_{j}$ the midpoint of edge $e_{j}$. This, together with Assumption 2.2, implies that both $a_{\mathrm{RT}}^{1 j}$ and $a_{\mathrm{RT}}^{2 j}$ are constants independent of the mesh size $h$. It follows from (3.16) and (3.17) that

$$
\gamma_{\mathrm{RT}}^{i j}=\frac{1}{h^{2}|K|}\left(\left(\phi_{\mathrm{RT}}^{i}, \phi_{\mathrm{RT}}^{j}\right)_{0, K}-\sum_{k=1}^{3}\left(a_{\mathrm{RT}}^{j k} \phi_{\mathrm{RT}}^{i}+a_{\mathrm{RT}}^{i k} \phi_{\mathrm{RT}}^{j}, \mathbf{x}-\mathbf{p}_{k}\right)_{0, K}+\sum_{k, l=1}^{3} a_{\mathrm{RT}}^{i k} a_{\mathrm{RT}}^{j l}\left(\mathbf{x}-\mathbf{p}_{k}, \mathbf{x}-\mathbf{p}_{l}\right)_{0, K}\right) .
$$

By the definition of $\phi_{\mathrm{RT}}^{i}$ and Assumption 2.2, for any $1 \leq i, j \leq 2,1 \leq k, l \leq 3$,

$$
\frac{1}{h^{2}|K|}\left(\phi_{\mathrm{RT}}^{i}, \phi_{\mathrm{RT}}^{j}\right)_{0, K}, \frac{1}{h^{2}|K|}\left(\phi_{\mathrm{RT}}^{j}, \mathbf{x}-\mathbf{p}_{k}\right)_{0, K}, \frac{1}{h^{2}|K|}\left(\mathbf{x}-\mathbf{p}_{k}, \mathbf{x}-\mathbf{p}_{l}\right)_{0, K}
$$

are constants independent of $h$. Thus, constants $\gamma_{\mathrm{RT}}^{i j}$ in (3.16) are also independent of the mesh size $h$, which completes the proof.

The following lemma presents the Taylor expansion of the interpolation error of the RT element for any quadratic polynomials. 
Lemma 3.3. For any $w \in P_{2}(K, \mathbb{R})$,

$$
\begin{aligned}
\left\|\left(I-\Pi_{\mathrm{RT}}\right) \nabla w\right\|_{0, K}^{2}= & h^{2}\left(\frac{\gamma_{\mathrm{RT}}^{11}}{4}\left\|\partial_{x_{1} x_{1}} w-\partial_{x_{2} x_{2}} w\right\|_{0, K}^{2}+\gamma_{\mathrm{RT}}^{22}\left\|\partial_{x_{1} x_{2}} w\right\|_{0, K}^{2}\right. \\
& \left.+\gamma_{\mathrm{RT}}^{12} \int_{K}\left(\partial_{x_{1} x_{1}} w-\partial_{x_{2} x_{2}} w\right) \partial_{x_{1} x_{2}} w d x\right),
\end{aligned}
$$

where $\gamma_{\mathrm{RT}}^{11} \gamma_{\mathrm{RT}}^{12}$ and $\gamma_{\mathrm{RT}}^{22}$ in (3.16) are independent constant of the mesh size $h$.

Proof. For any $w \in P_{2}(K, \mathbb{R})$, since $\nabla P_{2}\left(K, \mathbb{R}^{2}\right)=\mathrm{RT}\left(K, \mathbb{R}^{2}\right)+\operatorname{span}\left\{\phi_{\mathrm{RT}}^{1}, \phi_{\mathrm{RT}}^{2}\right\}$,

$$
\left(I-\Pi_{\mathrm{RT}}\right) \nabla w=a_{1}\left(I-\Pi_{\mathrm{RT}}\right) \phi_{\mathrm{RT}}^{1}+a_{2}\left(I-\Pi_{\mathrm{RT}}\right) \phi_{\mathrm{RT}}^{2}
$$

with the coefficients $a_{i}$ to be determined. For any vector $v=\left(v_{1}, v_{2}\right)$, define

$$
D_{1} v=\partial_{x_{1}} v_{1}-\partial_{x_{2}} v_{2}, \quad D_{2} v=\partial_{x_{2}} v_{1}+\partial_{x_{1}} v_{2} \text {. }
$$

Note that $D_{i} \phi_{\mathrm{RT}}^{j}=2 \delta_{i j}$ and $D_{i} \mathbf{x}=0$ for any $1 \leq i, j \leq 2$. By applying the operators $D_{1}$ and $D_{2}$ to the both sides of (3.19),

$$
a_{1}=\frac{1}{2}\left(\partial_{x_{1} x_{1}}-\partial_{x_{2} x_{2}}\right) w, \quad a_{2}=\partial_{x_{1} x_{2}} w
$$

A substitution of (3.20) to (3.19) leads to

$$
\left(I-\Pi_{\mathrm{RT}}\right) \nabla w=\frac{\left(\partial_{x_{1} x_{1}} w-\partial_{x_{2} x_{2}} w\right)}{2}\left(I-\Pi_{\mathrm{RT}}\right) \phi_{\mathrm{RT}}^{1}+\partial_{x_{1} x_{2}} w\left(I-\Pi_{\mathrm{RT}}\right) \phi_{\mathrm{RT}}^{2} .
$$

It follows from (3.16) and (3.21) that

$$
\begin{aligned}
\left\|\left(I-\Pi_{\mathrm{RT}}\right) \nabla w\right\|_{0, K}^{2}= & h^{2}\left(\frac{\gamma_{\mathrm{RT}}^{11}}{4}\left\|\partial_{x_{1} x_{1}} w-\partial_{x_{2} x_{2}} w\right\|_{0, K}^{2}+\gamma_{\mathrm{RT}}^{22}\left\|\partial_{x_{1} x_{2}} w\right\|_{0, K}^{2}\right. \\
& \left.+\gamma_{\mathrm{RT}}^{12} \int_{K}\left(\partial_{x_{1} x_{1}} w-\partial_{x_{2} x_{2}} w\right) \partial_{x_{1} x_{2}} w d x\right),
\end{aligned}
$$

which completes the proof.

For a smooth enough function $u$, to obtain a fourth-order accurate expansion of the interpolation error $\left(I-\Pi_{\mathrm{RT}}\right) \nabla u$, an orthogonal property as analyzed in Lemma 3.4 is needed. To this end, introduce the canonical interpolation of the Morley element in [26, 27]: let $\left.\Pi_{M} v\right|_{K} \in P_{2}(K, \mathbb{R})$ with

$$
\Pi_{\mathrm{M} v} v\left(\boldsymbol{p}_{i}\right)=v\left(\boldsymbol{p}_{i}\right), \quad \int_{e_{i}} \frac{\partial \Pi_{\mathrm{M}} v}{\partial \mathbf{n}} d s=\int_{e_{i}} \frac{\partial v}{\partial \mathbf{n}} d s \quad 1 \leq i \leq 3 .
$$

It follows that

$$
\int_{e} \nabla\left(I-\Pi_{M}\right) v d s=0, \quad \int_{K} \nabla^{2}\left(I-\Pi_{M}\right) v d x=0 \text { for any edge } e \text { and any element } K \text {. }
$$

For any element $K$, there exists the following error estimates for the interpolation error [26]

$$
\left|\left(I-\Pi_{\mathrm{M}}\right) v\right|_{m, K} \lesssim h^{3-m}|v|_{3, K}, \forall 0 \leq m \leq 3 .
$$


Lemma 3.4. Under the Assumption 2.2, for any $u \in H^{4}(\Omega, \mathbb{R})$, it holds that

$$
\left|\sum_{K \in \mathcal{T}_{h}}\left(\left(I-\Pi_{\mathrm{RT}}\right) \nabla \Pi_{\mathrm{M}} u,\left(I-\Pi_{\mathrm{RT}}\right) \nabla\left(I-\Pi_{\mathrm{M}}\right) u\right)_{0, K}\right| \lesssim h^{4}|u|_{4, \Omega}^{2}
$$

Proof. By the definition of the interpolations $\Pi_{\mathrm{RT}}$ and $\Pi_{\mathrm{M}}$,

$$
\Pi_{\mathrm{RT}} \nabla\left(I-\Pi_{\mathrm{M}}\right) u=0 .
$$

It remains to prove that

$$
\left|\sum_{K \in \mathcal{T}_{h}}\left(\left(I-\Pi_{\mathrm{RT}}\right) \nabla \Pi_{\mathrm{M}} u, \nabla\left(I-\Pi_{\mathrm{M}}\right) u\right)_{0, K}\right| \lesssim h^{4}|u|_{4, \Omega}^{2}
$$

Let $\Pi_{P_{3}}$ be the Larange interpolation operator of the $H^{1}$ conforming $P_{3}$ element, which leads to the following decomposition:

$$
\begin{aligned}
& \sum_{K \in \mathcal{T}_{h}}\left(\left(I-\Pi_{\mathrm{RT}}\right) \nabla \Pi_{\mathrm{M}} u, \nabla\left(I-\Pi_{\mathrm{M}}\right) u\right)_{0, K} \\
= & \sum_{K \in \mathcal{T}_{h}}\left(\left(I-\Pi_{\mathrm{RT}}\right) \nabla \Pi_{\mathrm{M}} \Pi_{\mathrm{P}_{3}} u, \nabla\left(I-\Pi_{\mathrm{M}}\right) \Pi_{\mathrm{P}_{3}} u\right)_{0, K} \\
& +\sum_{K \in \mathcal{T}_{h}}\left(\left(I-\Pi_{\mathrm{RT}}\right) \nabla \Pi_{\mathrm{M}}\left(I-\Pi_{\mathrm{P}_{3}}\right) u, \nabla\left(I-\Pi_{\mathrm{M}}\right) \Pi_{\mathrm{P}_{3}} u\right)_{0, K} \\
& +\sum_{K \in \mathcal{T}_{h}}\left(\left(I-\Pi_{\mathrm{RT}}\right) \nabla \Pi_{\mathrm{M}} u, \nabla\left(I-\Pi_{\mathrm{M}}\right)\left(I-\Pi_{\mathrm{P}_{3}}\right) u\right)_{0, K} \cdot
\end{aligned}
$$

Since $\left|\left(I-\Pi_{\mathrm{P}_{3}}\right) u\right|_{i, \Omega} \lesssim h^{4-i}|u|_{4, \Omega}$ for $0 \leq i \leq 3$, the second and third terms on the right-hand side of (3.25) can be estimated as, respectively,

$$
\begin{aligned}
& \left.\mid \sum_{K \in \mathcal{T}_{h}}\left(I-\Pi_{\mathrm{RT}}\right) \nabla \Pi_{\mathrm{M}}\left(I-\Pi_{\mathrm{P}_{3}}\right) u, \nabla\left(I-\Pi_{\mathrm{M}}\right) \Pi_{\mathrm{P}_{3}} u\right)\left._{0, K}\left|\lesssim h^{4}\right| u\right|_{4, \Omega} ^{2}, \\
& \left.\quad \mid \sum_{K \in \mathcal{T}_{h}}\left(I-\Pi_{\mathrm{RT}}\right) \nabla \Pi_{\mathrm{M}} u, \nabla\left(I-\Pi_{\mathrm{M}}\right)\left(I-\Pi_{\mathrm{P}_{3}}\right) u\right)\left._{0, K}\left|\lesssim h^{4}\right| u\right|_{4, \Omega} ^{2} .
\end{aligned}
$$

To analyze the first term on the right-hand side of (3.25), let $v_{h}=\Pi_{\mathrm{M}} \Pi_{\mathrm{P}_{3}} u$. Since $\operatorname{div}_{h}\left(\nabla_{h} v_{h}\right)$ is a piecewise constant, an integration by parts implies that

$$
\left.\operatorname{div}\left(I-\Pi_{\mathrm{RT}}\right) \nabla v_{h}\right|_{K}=\frac{1}{|K|} \int_{\partial K}\left(I-\Pi_{\mathrm{RT}}\right)\left(\nabla v_{h}\right) \cdot \mathbf{n} d s=0
$$

for any element $K \in \mathcal{T}_{h}$. Consequently,

$$
\left(\left(I-\Pi_{\mathrm{RT}}\right) \nabla v_{h}, \nabla\left(I-\Pi_{\mathrm{M}}\right) \Pi_{\mathrm{P}_{3}} u\right)_{0, K}=\sum_{i=1}^{3} \int_{e_{i}}\left(I-\Pi_{\mathrm{M}}\right) \Pi_{\mathrm{P}_{3}} u\left(\left(I-\Pi_{\mathrm{RT}}\right) \nabla v_{h} \cdot \mathbf{n}\right) d s .
$$


Given edge $e_{i}$ of element $K$, recall that $\mathbf{n}_{i}$ and $\mathbf{t}_{i}$ are the unit outward normal vector and the unit tangent vector, respectively. Note that $\frac{\partial^{2} v_{h}}{\partial \mathbf{t}_{i} \partial \mathbf{n}_{i}} \in P_{0}(K, \mathbb{R})$ and

$$
\left.\left(I-\Pi_{\mathrm{RT}}\right) \nabla v_{h} \cdot \mathbf{n}_{i}\right|_{e_{i}}=\frac{\partial v_{h}}{\partial \mathbf{n}_{i}}-\frac{\partial v_{h}}{\partial \mathbf{n}_{i}}\left(\mathbf{m}_{i}\right)=\left|e_{i}\right| \frac{\partial^{2} v_{h}}{\partial \mathbf{t}_{i} \partial \mathbf{n}_{i}}\left(\psi_{i-1}-\frac{1}{2}\right) .
$$

with $\mathbf{m}_{i}$ the midpoint of edge $e_{i}$ and $\psi_{i}$ the barycenter coordinates. It follows from (3.27) and (3.28) that

$$
\left(\left(I-\Pi_{\mathrm{RT}}\right) \nabla v_{h}, \nabla\left(I-\Pi_{\mathrm{M}}\right) \Pi_{\mathrm{P}_{3}} u\right)_{0, K}=\sum_{i=1}^{3}\left|e_{i}\right| \frac{\partial^{2} v_{h}}{\partial \mathbf{t}_{i} \partial \mathbf{n}_{i}} \int_{e_{i}}\left(I-\Pi_{\mathrm{M}}\right) \Pi_{\mathrm{P}_{3}} u\left(\psi_{i-1}-\frac{1}{2}\right) d s .
$$

Since $\Pi_{\mathrm{M}} \Pi_{\mathrm{P}_{3}} u \in P_{2}(K, \mathbb{R})$, the Taylor expansion indicates that

$$
\begin{aligned}
\left.\left(I-\Pi_{\mathrm{M}}\right) \Pi_{\mathrm{P}_{3}} u\right|_{e_{i}=} & \left(I-\Pi_{\mathrm{M}}\right) \Pi_{\mathrm{P}_{3}} u\left(\mathbf{m}_{i}\right)+\left|e_{i}\right| \frac{\partial\left(I-\Pi_{\mathrm{M}}\right) \Pi_{\mathrm{P}_{3}} u}{\partial \mathbf{t}_{i}}\left(\mathbf{m}_{i}\right)\left(\psi_{i-1}-\frac{1}{2}\right) \\
& +\frac{\left|e_{i}\right|^{2}}{2} \frac{\partial^{2}\left(I-\Pi_{\mathrm{M}}\right) \Pi_{\mathrm{P}_{3}} u}{\partial \mathbf{t}_{i}^{2}}\left(\mathbf{m}_{i}\right)\left(\psi_{i-1}-\frac{1}{2}\right)^{2} \\
& +\frac{\left|e_{i}\right|^{3}}{6} \frac{\partial^{3} \Pi_{\mathrm{P}_{3}} u}{\partial \mathbf{t}_{i}^{3}}\left(\mathbf{m}_{i}\right)\left(\psi_{i-1}-\frac{1}{2}\right)^{3}
\end{aligned}
$$

The Taylor expansion of $\frac{\partial\left(I-\Pi_{\mathrm{M}}\right) \Pi_{\mathrm{P}_{3}} u}{\partial \mathrm{t}_{i}}$ reads

$$
\begin{aligned}
\left.\frac{\partial\left(I-\Pi_{\mathrm{M}}\right) \Pi_{\mathrm{P}_{3}} u}{\partial \mathbf{t}_{i}}\right|_{e_{i}}= & \frac{\partial\left(I-\Pi_{\mathrm{M}}\right) \Pi_{\mathrm{P}_{3}} u}{\partial \mathbf{t}_{i}}\left(\mathbf{m}_{i}\right)+\left|e_{i}\right| \frac{\partial^{2}\left(I-\Pi_{\mathrm{M}}\right) \Pi_{\mathrm{P}_{3}} u}{\partial \mathbf{t}_{i}^{2}}\left(\mathbf{m}_{i}\right)\left(\psi_{i-1}-\frac{1}{2}\right) \\
& +\frac{\left|e_{i}\right|^{2}}{2} \frac{\partial^{3} \Pi_{\mathrm{P}_{3}} u}{\partial \mathbf{t}_{i}^{3}}\left(\mathbf{m}_{i}\right)\left(\psi_{i-1}-\frac{1}{2}\right)^{2} .
\end{aligned}
$$

By (3.23) and the fact that $\int_{e_{i}} \psi_{i-1}-\frac{1}{2} d s=0$ and $\int_{e_{i}}\left(\psi_{i-1}-\frac{1}{2}\right)^{2} d s=\frac{\left|e_{i}\right|}{12}$,

$$
0=\int_{e_{i}} \frac{\partial\left(I-\Pi_{\mathrm{M}}\right) \Pi_{\mathrm{P}_{3}} u}{\partial \mathbf{t}_{i}} d s=\left|e_{i}\right| \frac{\partial\left(I-\Pi_{\mathrm{M}}\right) \Pi_{\mathrm{P}_{3}} u}{\partial \mathbf{t}_{i}}\left(\mathbf{m}_{i}\right)+\frac{\left|e_{i}\right|^{3}}{24} \frac{\partial^{3} \Pi_{\mathrm{P}_{3}} u}{\partial \mathbf{t}_{i}^{3}} .
$$

Thus,

$$
\frac{\partial\left(I-\Pi_{\mathrm{M}}\right) \Pi_{\mathrm{P}_{3}} u}{\partial \mathbf{t}_{i}}\left(\mathbf{m}_{i}\right)=-\frac{\left|e_{i}\right|^{2}}{24} \frac{\partial^{3} \Pi_{\mathrm{P}_{3}} u}{\partial \mathbf{t}_{i}^{3}} .
$$

Since $\int_{e_{i}}\left(\psi_{i-1}-\frac{1}{2}\right)^{j} d s=0$ for $j=1$ and 3 , a substitution of (3.31) into (3.30) yields

$$
\int_{e_{i}}\left(I-\Pi_{\mathrm{M}}\right) \Pi_{\mathrm{P}_{3}} u\left(\psi_{i-1}-\frac{1}{2}\right) d s=-\frac{\left|e_{i}\right|^{4}}{720} \frac{\partial^{3} \Pi_{\mathrm{P}_{3}} u}{\partial \mathbf{t}_{i}^{3}} .
$$

By the above equation and (3.29),

$$
\left(\left(I-\Pi_{\mathrm{RT}}\right) \nabla v_{h}, \nabla\left(I-\Pi_{\mathrm{M}}\right) \Pi_{\mathrm{P}_{3}} u\right)_{0, K}=-\sum_{i=1}^{3} \frac{\left|e_{i}\right|^{4}}{720} \int_{e_{i}} \frac{\partial^{2} v_{h}}{\partial \mathbf{t}_{i} \partial \mathbf{n}_{i}} \frac{\partial^{3} \Pi_{\mathrm{P}_{3}} u}{\partial \mathbf{t}_{i}^{3}} d s .
$$


Since $\Pi_{P_{3}} u$ is continuous on interior edges, and $\left.\mathbf{t}_{i}\right|_{K_{1}}=-\left.\mathbf{t}_{i}\right|_{K_{2}}$ and $\left.\mathbf{n}_{i}\right|_{K_{1}}=-\left.\mathbf{n}_{i}\right|_{K_{2}}$, a summation over all the elements leads to

$$
\sum_{K \in \mathcal{T}_{h}}\left(\left(I-\Pi_{\mathrm{RT}}\right) \nabla v_{h}, \nabla\left(I-\Pi_{\mathrm{M}}\right) \Pi_{\mathrm{P}_{3}} u\right)_{0, K}=-\sum_{e \in \mathcal{E}_{h}} \frac{|e|^{4}}{720} \int_{e}\left[\frac{\partial^{2}\left(v_{h}-u\right)}{\partial \mathbf{t}_{e} \partial \mathbf{n}_{e}}\right] \frac{\partial^{3} \Pi_{\mathrm{P}_{3}} u}{\partial \mathbf{t}_{e}^{3}} d s .
$$

By the trace inequality, the triangle inequality and the above equation,

$$
\left|\sum_{K \in \mathcal{T}_{h}}\left(\left(I-\Pi_{\mathrm{RT}}\right) \nabla v_{h}, \nabla\left(I-\Pi_{\mathrm{M}}\right) \Pi_{\mathrm{P}_{3}} u\right)_{0, K}\right| \lesssim h^{4}|u|_{3, \Omega}^{2} .
$$

A substitution of (3.26) and (3.32) into (3.25) leads to

$$
\left|\sum_{K \in \mathcal{T}_{h}}\left(\left(I-\Pi_{\mathrm{RT}}\right) \nabla \Pi_{\mathrm{M}} u, \nabla\left(I-\Pi_{\mathrm{M}}\right) u\right)_{0, K}\right| \lesssim h^{4}|u|_{4, \Omega}^{2}
$$

which completes the proof.

Thanks to Lemmas 3.3 and 3.4 , there exists the following fourth-order accurate expansion of the term $\left\|\left(I-\Pi_{\mathrm{RT}}\right) \nabla u\right\|_{0, \Omega}^{2}$ in Theorem 3.1 .

Lemma 3.5. For any $u \in H^{4}(\Omega, \mathbb{R})$,

$$
\begin{aligned}
\left\|\left(I-\Pi_{\mathrm{RT}}\right) \nabla u\right\|_{0, \Omega}^{2}= & h^{2}\left(\frac{\gamma_{\mathrm{RT}}^{11}}{4}\left\|\partial_{x_{1} x_{1}} u-\partial_{x_{2} x_{2}} u\right\|_{0, \Omega}^{2}+\gamma_{\mathrm{RT}}^{22}\left\|\partial_{x_{1} x_{2}} u\right\|_{0, \Omega}^{2}\right. \\
& \left.+\gamma_{\mathrm{RT}}^{12} \int_{\Omega}\left(\partial_{x_{1} x_{1}} u-\partial_{x_{2} x_{2}} u\right) \partial_{x_{1} x_{2}} u d x\right)+O\left(h^{4}|u|_{4, \Omega}^{2}\right) .
\end{aligned}
$$

3.3. Refined analysis of $I_{\mathrm{CR}}=2\left(\left(I-\Pi_{\mathrm{RT}}\right) \nabla u, \sigma_{\mathrm{RT}}^{\lambda u}-\nabla_{h} u_{\mathrm{CR}}^{\lambda \Pi_{h}^{0} u}\right)-2 \lambda\left(u-\Pi_{\mathrm{CR}} u, u\right)$. For this term, a direct use of the Cauchy-Schwarz inequality and the Taylor expansions of interpolation errors only leads to a suboptimal expansion since the second term of $I_{\mathrm{CR}}$ is essentially a consistency error and only admits a third order convergence which can not be improved. The idea here is to explore the relation (2.20)

$$
\left.2\left(\sigma_{\mathrm{RT}}^{\lambda u}-\nabla_{h} u_{\mathrm{CR}}^{\lambda \Pi_{h}^{0} u}\right)\right|_{K}=-\lambda \Pi_{K}^{0} u\left(\mathbf{x}-\mathbf{M}_{K}\right)
$$

between the RT element and the CR element for any $K \in \mathcal{T}_{h}$, and decompose the first term of $I_{\mathrm{CR}}$ into two terms: one cancels the second term of $I_{\mathrm{CR}}$ and the other one has an asymptotic expansion. To this end, one needs the following crucial superconvergence of the inner product of the errors of the canonical interpolation of the CR element and the piecewise constant $L^{2}$ projection.

Lemma 3.6. If two adjacent elements $K_{1}$ and $K_{2}$ form a parallelogram. For any $w \in P_{2}\left(K_{1} \cup K_{2}, \mathbb{R}\right)$ and $v \in P_{1}\left(K_{1} \cup K_{2}, \mathbb{R}\right)$, it holds that

$$
\left(w-\Pi_{\mathrm{CR}} w, v-\Pi_{h}^{0} v\right)_{0, K_{1} \cup K_{2}}=0 .
$$

Furthermore, under the Assumption 2.2 for any $u \in H^{3}(\Omega, \mathbb{R})$,

$$
\left|\left(u-\Pi_{\mathrm{CR}} u, u-\Pi_{h}^{0} u\right)\right| \lesssim h^{4}|u|_{3, \Omega}^{2} .
$$


Proof. In order to derive an expression of the error $w-\Pi_{\mathrm{CR}} w$, define three basis functions

$$
\phi_{\mathrm{CR}}^{i}=\left(2 \psi_{i-1}-1\right)\left(2 \psi_{i+1}-1\right)-\frac{2}{3} \psi_{i}+\frac{1}{3}, \quad 1 \leq i \leq 3,
$$

with the barycentric coordinates $\left\{\psi_{i}\right\}_{i=1}^{3}$ of element $K$. These basis functions satisfy that

$$
\int_{e_{j}} \phi_{\mathrm{CR}}^{i} d s=0, \quad \forall 1 \leq i, j \leq 3 .
$$

This implies that these functions are bubble functions of the canonical interpolation operator $\Pi_{\mathrm{CR}}$ of the $\mathrm{CR}$ element. Thus, for any quadratic polynomial $w$,

$$
\left(I-\Pi_{\mathrm{CR}}\right) w=\sum_{i=1}^{3} c_{i} \phi_{\mathrm{CR}}^{i}
$$

To compute the coefficients $c_{i}, i=1,2,3$, recall the gradient $\nabla \psi_{i}=-\frac{\mathbf{n}_{i}}{d_{i}}$ of the barycenter coordinate $\psi_{i}$ from (2.1). This gives

$$
\frac{\partial^{2}}{\partial \mathbf{t}_{j}^{2}} \phi_{\mathrm{CR}}^{i}=-\frac{8}{\left|e_{i}\right|^{2}} \delta_{i j}
$$

Thus, taking second order tangential derivatives on both sides of (3.36) yields

$$
c_{i}=-\frac{\left|e_{i}\right|^{2}}{8|K|} \int_{K} \frac{\partial^{2} w}{\partial \mathbf{t}_{i}^{2}} d x, \quad 1 \leq i \leq 3
$$

This and (3.36) lead to an expression of the error $w-\Pi_{\mathrm{CR}} w$ as follows

$$
\left(I-\Pi_{\mathrm{CR}}\right) w=-\frac{1}{8} \sum_{i=1}^{3}\left|e_{i}\right|^{2} \frac{\partial^{2} w}{\partial \mathbf{t}_{i}^{2}} \phi_{\mathrm{CR}}^{i}
$$

For any linear polynomial $v$, it holds that

$$
\left(I-\Pi_{K}^{0}\right) v=\sum_{i=1}^{3} v\left(p_{i}\right)\left(\psi_{i}-\frac{1}{3}\right), \quad\left(\phi_{\mathrm{CR}}^{i}, \psi_{j}-\frac{1}{3}\right)_{0, K}=\left\{\begin{array}{cl}
\frac{4}{135}|K|, & \text { if } i=j \\
\frac{-2}{135}|K|, & \text { if } i \neq j
\end{array} .\right.
$$

A combination of (3.37) and (3.38) leads to

$$
\begin{aligned}
\left(w-\Pi_{\mathrm{CR}} w, v-\Pi_{K}^{0} v\right)_{0, K} & =-\frac{1}{540} \sum_{i=1}^{3} \frac{\partial^{2} w}{\partial \mathbf{t}_{i}^{2}}\left|e_{i}\right|^{2}|K|\left(2 v\left(\boldsymbol{p}_{i}\right)-v\left(\boldsymbol{p}_{i-1}\right)-v\left(\boldsymbol{p}_{i+1}\right)\right) \\
& =-\frac{1}{270} \sum_{i=1}^{3} \frac{\partial^{2} w}{\partial \mathbf{t}_{i}^{2}}\left|e_{i}\right|^{2}|K| \nabla v \cdot{\overrightarrow{\boldsymbol{m}_{i} \boldsymbol{p}_{i}}} .
\end{aligned}
$$

Suppose $\boldsymbol{p}_{K_{1}}$ and $\boldsymbol{p}_{K_{2}}$ are the vertices of the element $K_{1}$ and $K_{2}$, respectively, and the opposite edge $e_{K_{1}}$ to $\boldsymbol{p}_{K_{1}}$ in $K_{1}$ is parallel to the opposite edge $e_{K_{2}}$ to $\boldsymbol{p}_{K_{2}}$ in $K_{2}$. Let $\boldsymbol{m}_{K_{1}}$ and 
$\boldsymbol{m}_{K_{2}}$ be the midpoints of $e_{K_{1}}$ and $e_{K_{2}}$, respectively. Since the elements $K_{1}$ and $K_{2}$ form a parallelogram,

$$
\left|K_{1}\right|=\left|K_{2}\right|, \quad \overrightarrow{\boldsymbol{m}_{K_{1}} \boldsymbol{p}_{K_{1}}}=-\overrightarrow{\boldsymbol{m}_{K_{2}} \boldsymbol{p}_{K_{2}}} .
$$

Since $\nabla^{2} w$ and $\nabla v$ are constant in $K_{1} \cup K_{2}$, a combination of (3.39) and (3.40) yields

$$
\left(w-\Pi_{\mathrm{CR}} w, v-\Pi_{h}^{0} v\right)_{0, K_{1} \cup K_{2}}=0,
$$

which completes the proof for (3.34).

The partition $\mathcal{T}_{h}$ of domain $\Omega$ includes the set of parallelograms $\mathcal{N}_{1}$ and the set of a few remaining boundary triangles $\mathcal{N}_{2}$. Let $\kappa=\left|\mathcal{N}_{2}\right|$ denote the number of the elements in $\mathcal{N}_{2}$.

$$
\left(u-\Pi_{\mathrm{CR}} u, u-\Pi_{h}^{0} u\right)=\sum_{K \in \mathcal{N}_{1}}\left(u-\Pi_{\mathrm{CR}} u, u-\Pi_{h}^{0} u\right)_{0, K}+\sum_{K \in \mathcal{N}_{2}}\left(u-\Pi_{\mathrm{CR}} u, u-\Pi_{h}^{0} u\right)_{0, K}
$$

A direct application of the Bramble-Hilbert Lemma to (3.34) leads to

$$
\left|\sum_{K \in \mathcal{N}_{1}}\left(u-\Pi_{\mathrm{CR}} u, u-\Pi_{h}^{0} u\right)_{0, K}\right| \lesssim h^{4}|u|_{3, \Omega}^{2} .
$$

For any element $K \in \mathcal{N}_{2}$, it follows from the error estimates of (2.7), (2.17) and the triangle inequality that

$$
\left|\left(u-\Pi_{\mathrm{CR}} u, u-\Pi_{h}^{0} u\right)_{0, K}\right| \lesssim h^{4}|u|_{2, K}|u|_{1, \infty},
$$

consequently,

$$
\left|\sum_{K \in \mathcal{N}_{2}}\left(u-\Pi_{\mathrm{CR}} u, u-\Pi_{h}^{0} u\right)_{0, K}\right| \lesssim \sqrt{\kappa} h^{4}|u|_{3, \Omega}^{2} .
$$

A substitution of (3.42) and (3.43) into (3.41) leads to (3.35) and completes the proof.

The following lemma shows an asymptotic expansion of $I_{\mathrm{CR}}$ in Theorem 3.1 .

Lemma 3.7. Suppose that $u \in H^{3}(\Omega, \mathbb{R})$. Under the Assumption 2.2.

$$
I_{\mathrm{CR}}=-\frac{\lambda^{2} H^{2}}{72}+O\left(h^{4}|u|_{3, \Omega}^{2}\right)
$$

Proof. Recall the definition of $I_{\mathrm{CR}}$ in 3.9

$$
I_{\mathrm{CR}}=2\left(\left(I-\Pi_{\mathrm{RT}}\right) \nabla u, \sigma_{\mathrm{RT}}^{\lambda u}-\nabla_{h} u_{\mathrm{CR}}^{\lambda \Pi_{h}^{0} u}\right)-2 \lambda\left(u-\Pi_{\mathrm{CR}} u, u\right) .
$$

For the first term on the right-hand side, it follows from the relation (2.20) between the CR element and the RT element that

$2\left(\left(I-\Pi_{\mathrm{RT}}\right) \nabla u, \sigma_{\mathrm{RT}}^{\lambda u}-\nabla_{h} u_{\mathrm{CR}}^{\lambda \Pi_{h}^{0} u}\right)=-\lambda \sum_{K \in \mathcal{T}_{h}}\left(\left(\nabla u, \Pi_{K}^{0} u\left(\mathbf{x}-\mathbf{M}_{K}\right)\right)_{0, K}-\left(\Pi_{\mathrm{RT}} \nabla u, \Pi_{K}^{0} u\left(\mathbf{x}-\mathbf{M}_{K}\right)\right)_{0, K}\right)$.

One key for the analysis is to decompose the first term on the right-hand side of the above equation. Indeed, since $\left.\nabla_{h} \Pi_{\mathrm{CR}} u\right|_{K} \in P_{0}\left(K, \mathbb{R}^{2}\right)$ and $\int_{K} \mathbf{x}-\mathbf{M}_{K} d x=0$, it holds that

$$
\left(\nabla u, \Pi_{K}^{0} u\left(\mathbf{x}-\mathbf{M}_{K}\right)\right)_{0, K}=\left(\nabla_{h}\left(u-\Pi_{\mathrm{CR}} u\right), \Pi_{K}^{0} u\left(\mathbf{x}-\mathbf{M}_{K}\right)\right)_{0, K}
$$


Thus, it follows from $\left.\left(\mathbf{x}-\mathbf{M}_{K}\right) \cdot \mathbf{n}\right|_{e} \in P_{0}(e, \mathbb{R})$ and an integration by parts that

$$
\left(\nabla u, \Pi_{K}^{0} u\left(\mathbf{x}-\mathbf{M}_{K}\right)\right)_{0, K}=-2\left(u-\Pi_{\mathrm{CR}} u, u\right)+2\left(u-\Pi_{\mathrm{CR}} u, u-\Pi_{K}^{0} u\right)_{0, K} \cdot
$$

Multiplying both side of the above equation by $\lambda$, there is a cancellation between the first term on the right-hand side of the above equation and the second term of $I_{\mathrm{CR}}$ while the second term on the right-hand side of the above equation can be bounded by the superconvergence analyzed in (3.35). This yields

$$
I_{\mathrm{CR}}=\lambda \sum_{K \in \mathcal{T}_{h}}\left(\Pi_{\mathrm{RT}} \nabla u, \Pi_{K}^{0} u\left(\mathbf{x}-\mathbf{M}_{K}\right)\right)_{0, K}+O\left(h^{4}|u|_{3, \Omega}^{2}\right)
$$

It remains to analyze the first term on the right hand side of the above equation. For the gradient $\nabla u$, its interpolation of the RT element reads

$$
\left.\Pi_{\mathrm{RT}} \nabla u\right|_{K}=\sum_{i=1}^{3} \frac{1}{2|K|} \int_{e_{i}} \frac{\partial u}{\partial n} d s\left(\mathbf{x}-\mathbf{p}_{i}\right)
$$

Since $\int_{K} \mathbf{x}-\mathbf{M}_{K} d x=0$,

$$
\left(\mathbf{x}-\mathbf{p}_{i}, \mathbf{x}-\mathbf{M}_{K}\right)=\left(\mathbf{x}-\mathbf{M}_{K}, \mathbf{x}-\mathbf{M}_{K}\right)=\frac{H^{2}|K|}{36}, \quad \forall 1 \leq i \leq 3 .
$$

This implies that

$$
\left(\Pi_{\mathrm{RT}} \nabla u, \Pi_{K}^{0} u\left(\mathbf{x}-\mathbf{M}_{K}\right)\right)_{0, K}=\frac{H^{2}}{72} \int_{\partial K} \Pi_{K}^{0} u \frac{\partial u}{\partial n} d s=\frac{H^{2}}{72}\left(\Pi_{K}^{0} u, \Delta u\right)_{0, K} \cdot
$$

By the orthogonal property of the constant $L^{2}$ projection operator $\Pi_{K^{\prime}}^{0}$

$$
\left(\Pi_{K}^{0} u, \Delta u\right)_{0, K}=(u, \Delta u)_{0, K}+\left(\Pi_{K}^{0} u-u, \Delta u-\Pi_{K}^{0} \Delta u\right)_{0, K} .
$$

Since $H^{2}=O\left(h^{2}\right)$, a combination of this, the error estimate (2.17) for the $L^{2}$ projection operator $\Pi_{K}^{0}$, and (3.45) yields

$$
\sum_{K \in \mathcal{T}_{h}}\left(\Pi_{\mathrm{RT}} \nabla u, \Pi_{K}^{0} u\left(\mathbf{x}-\mathbf{M}_{K}\right)\right)_{0, K}=\sum_{K \in \mathcal{T}_{h}} \frac{H^{2}}{72} \int_{K} u \Delta u d x+O\left(h^{4}|u|_{3, \Omega}^{2}\right) .
$$

Under the Assumption 2.2, the constant $H^{2}$ is the same on all elements. Since $\Delta u=-\lambda u$ and $\|u\|_{0, \Omega}=1$, a substitution of (3.46) into (3.44) leads to

$$
I_{\mathrm{CR}}=-\frac{\lambda^{2} H^{2}}{72}+O\left(h^{4}|u|_{3, \Omega}^{2}\right)
$$

which completes the proof. 
3.4. Error estimate of $I_{\mathrm{RT}}=2\left(\nabla u-\Pi_{\mathrm{RT}} \nabla u, \Pi_{\mathrm{RT}} \nabla u-\sigma_{\mathrm{RT}}^{\lambda u}\right)$. The fact that $\Pi_{\mathrm{RT}} \nabla u-\sigma_{\mathrm{RT}}^{\lambda u}$ is divergence free and admits the superconvergence (2.15) leads to the following optimal analysis of $I_{\mathrm{RT}}$.

Lemma 3.8. Suppose that $\left(\sigma_{\mathrm{RT}}^{\lambda u}, u_{\mathrm{RT}}^{\lambda u}\right)$ is the RT solution of the discrete source problem (2.13) and $u \in H^{\frac{7}{2}}(\Omega, \mathbb{R})$. Under the Assumption 2.2 .

$$
\left|I_{\mathrm{RT}}\right| \lesssim h^{4}|\ln h \| u|_{\frac{7}{2}, \Omega}^{2} .
$$

Proof. With $\tau_{h}=\Pi_{\mathrm{RT}} \nabla u-\sigma_{\mathrm{RT}}^{\lambda u}$, the superconvergence result of the RT element (2.15) leads to

$$
I_{\mathrm{RT}}=\left(\nabla u-\sigma_{\mathrm{RT}}^{\lambda u}, \tau_{h}\right)+\left\|\tau_{h}\right\|_{0, \Omega}^{2}=\left(\nabla u, \tau_{h}\right)-\left(\sigma_{\mathrm{RT}}^{\lambda u}, \tau_{h}\right)+O\left(h^{4}|\ln h \| u|_{\frac{7}{2}, \Omega}^{2}\right) .
$$

It follows from the integration by parts and Problem (2.13) that

$$
I_{\mathrm{RT}}=\sum_{K \in \mathcal{T}_{h}}\left(-\int_{K} u \operatorname{div} \tau_{h} d x+\int_{\partial K} u \tau_{h} \cdot \mathbf{n} d s\right)+\left(u_{\mathrm{RT}}^{\lambda u}, \operatorname{div} \tau_{h}\right)+O\left(h^{4}|\ln h \| u|_{\frac{7}{2}, \Omega}^{2}\right) .
$$

Since $\operatorname{div} \tau_{h}=0, \tau_{h}$ is $\mathrm{H}(\operatorname{div})$-conforming, and $u$ vanishes on the boundary $\partial \Omega$, this completes the proof.

3.5. Error estimate of $I_{\mathrm{CR}}^{1}=2\left(\nabla u-\Pi_{\mathrm{RT}} \nabla u, \nabla_{h} u_{\mathrm{CR}}^{\lambda \Pi_{h}^{0} u}-\nabla_{h} u_{\mathrm{CR}}\right)$. A direct combination of Cauchy-Schwarz inequality and the superclose property (3.2) of the CR element only yields a suboptimal analysis of $I_{\mathrm{CR}}^{1}$. The idea here is to further make use of the relation (2.20) between the CR element and the RT element and decompose it into three terms: a vanishing term, a fourth order term, and a remaining term. By using the commuting property of $\Pi_{C R}$, the discrete eigenvalue problem (2.4) and the discrete source problem (2.18), and fully exploring the properties of the projection operator $\Pi_{h}^{0}$ and the uniformity of the mesh, this remaining term can be in some sense transferred to a consistency error with respect to the nonconforming function $\Pi_{\mathrm{CR}} u-u_{\mathrm{CR}}^{\lambda \Pi_{h}^{0} u}$. This, in fact, leads to the following superconvergence result.

Lemma 3.9. Suppose Assumption 2.2 holds. Let $s_{h}=\Pi_{\mathrm{CR}} u-u_{\mathrm{CR}}^{\lambda \Pi_{h}^{0} u}$, it holds that

$$
\left|\left(\left(I-\Pi_{h}^{0}\right) s_{h},\left(I-\Pi_{h}^{0}\right) u_{\mathrm{CR}}\right)\right| \lesssim h^{4}|u|_{2, \Omega}^{2}
$$

with $\left(\lambda_{\mathrm{CR}}, u_{\mathrm{CR}}\right)$ an eigenpair of (2.4) by the CR element and $u_{\mathrm{CR}}^{\lambda \Pi_{h}^{0} u}$ the solution of (2.18).

Proof. The direct use of the error estimate of the piecewise constant $L^{2}$ projection and the usual Cauchy-Schwarz inequality can only derive a second order convergence for this inner product. The idea herein is to fully explore the properties of the projection operator $\Pi_{h}^{0}$ and the uniformity of the mesh and transfer it to a consistency error. Indeed, given $K \in \mathcal{T}_{h}$, since $s_{h},\left.u_{\mathrm{CR}}\right|_{K} \in P_{1}(K, \mathbb{R})$, there exist the following expansions

$$
\left.\left(I-\Pi_{h}^{0}\right) s_{h}\right|_{K}=\sum_{i=1}^{2} \frac{\partial s_{h}}{\partial x_{i}}\left(x_{i}-M_{i}\right),\left.\quad\left(I-\Pi_{h}^{0}\right) u_{\mathrm{CR}}\right|_{K}=\sum_{i=1}^{2} \frac{\partial u_{\mathrm{CR}}}{\partial x_{i}}\left(x_{i}-M_{i}\right),
$$


with $\mathbf{M}_{K}=\left(M_{1}, M_{2}\right)$ the centroid of element $K$. Let $a_{i j}=\frac{1}{|K|} \int_{K}\left(x_{i}-M_{i}\right)\left(x_{j}-M_{j}\right) d x$. Thus,

$$
\left(\left(I-\Pi_{h}^{0}\right) s_{h},\left(I-\Pi_{h}^{0}\right) u_{\mathrm{CR}}\right)_{0, K}=\sum_{i, j=1}^{2} a_{i j} \int_{K} \frac{\partial s_{h}}{\partial x_{i}} \frac{\partial u_{\mathrm{CR}}}{\partial x_{j}} d x .
$$

Since the mesh is uniform, the constant $a_{i j}$ is equal for all the elements of the mesh and is of $O\left(h^{2}\right)$. Together with the error estimate (2.7) of the CR element, this gives

$$
\left|a_{i j} \sum_{K \in \mathcal{T}_{h}} \int_{K} \frac{\partial s_{h}}{\partial x_{i}} \frac{\partial\left(u_{\mathrm{CR}}-u\right)}{\partial x_{j}} d x\right| \lesssim h^{4}|u|_{2, \Omega}^{2},
$$

where the following error estimate is employed

$$
\left\|s_{h}\right\|_{0, \Omega}+h\left\|\nabla_{h} s_{h}\right\|_{0, \Omega} \lesssim h^{2}|u|_{2, \Omega} .
$$

Therefore,

$$
\left(\left(I-\Pi_{h}^{0}\right) s_{h},\left(I-\Pi_{h}^{0}\right) u_{\mathrm{CR}}\right)=\sum_{i, j=1}^{2} a_{i j} \sum_{K \in \mathcal{T}_{h}} \int_{K} \frac{\partial s_{h}}{\partial x_{i}} \frac{\partial u}{\partial x_{j}} d x+O\left(h^{4}|u|_{2, \Omega}^{2}\right) .
$$

Here the term $\sum_{K \in \mathcal{T}_{h}} \int_{K} \frac{\partial s_{h}}{\partial x_{i}} \frac{\partial u}{\partial x_{j}} d x$ is essentially a consistency error. In fact, an integration by parts gives

$$
\sum_{K \in \mathcal{T}_{h}} \int_{K} \frac{\partial s_{h}}{\partial x_{i}} \frac{\partial u}{\partial x_{j}} d x=-\sum_{e \in \mathcal{E}^{i}} \int_{e}\left[s_{h}\right] \frac{\partial u}{\partial x_{j}} n_{i} d s+\left(s_{h}, \frac{\partial^{2} u}{\partial x_{i} \partial x_{j}}\right) .
$$

Since $s_{h} \in V_{\mathrm{CR}}$ and $\int_{e}\left[s_{h}\right] d s=0$ for any $e \in \mathcal{E}^{i}$, the first term on the right-hand side of the above equation can be estimated as

$$
\left|\sum_{e \in \mathcal{E}^{i}} \int_{e}\left[s_{h}\right] \frac{\partial u}{\partial x_{j}} n_{i} d s\right|=\left|\sum_{e \in \mathcal{E}^{i}} \int_{e}\left[\left(I-\Pi_{e}^{0}\right) s_{h}\right]\left(I-\Pi_{e}^{0}\right) \frac{\partial u}{\partial x_{j}} n_{i} d s\right| \lesssim h^{2}|u|_{2, \Omega}^{2} .
$$

By the error estimate of (3.48), the second term on the right-hand side of (3.49) can be bounded as

$$
\left(s_{h}, \frac{\partial^{2} u}{\partial x_{i} \partial x_{j}}\right) \lesssim h^{2}|u|_{2, \Omega}^{2} .
$$

A summary of these estimates completes the proof.

Lemma 3.10. Let $\left(\lambda_{\mathrm{CR}}, u_{\mathrm{CR}}\right)$ be an eigenpair of (2.4) by the CR element and $u_{\mathrm{CR}}^{\lambda \Pi_{h}^{0} u}$ be the solution of (2.18). Suppose $u \in H^{\frac{7}{2}}(\Omega, \mathbb{R})$. Under the Assumption 2.2.

$$
\left|I_{\mathrm{CR}}^{1}\right| \lesssim h^{4}|\ln h|^{\frac{1}{2}}|u|_{\frac{7}{2}, \Omega}^{2}
$$


Proof. By the relation (2.20) between the CR element and the RT element, there is the following decomposition:

$$
\begin{aligned}
I_{\mathrm{CR}}^{1}= & 2\left(\nabla u-\nabla_{h} u_{\mathrm{CR}}^{\lambda \Pi_{h}^{0} u}, \nabla_{h} u_{\mathrm{CR}}^{\lambda \Pi_{h}^{0} u}-\nabla_{h} u_{\mathrm{CR}}\right) \\
& +\lambda \sum_{K \in \mathcal{T}_{h}}\left(\Pi_{K}^{0} u\left(\mathbf{x}-\mathbf{M}_{K}\right), \nabla_{h} u_{\mathrm{CR}}^{\lambda \Pi_{h}^{0} u}-\nabla_{h} u_{\mathrm{CR}}\right)_{0, K} \\
& +2\left(\sigma_{\mathrm{RT}}^{\lambda u}-\Pi_{\mathrm{RT}} \nabla u, \nabla_{h} u_{\mathrm{CR}}^{\lambda \Pi_{h}^{0} u}-\nabla_{h} u_{\mathrm{CR}}\right) .
\end{aligned}
$$

Since $\int_{K} \mathbf{x}-\mathbf{M}_{K} d x=0$ and $\nabla_{h} u_{\mathrm{CR}}^{\lambda \Pi_{h}^{0} u}-\left.\nabla_{h} u_{\mathrm{CR}}\right|_{K} \in P_{0}\left(K, \mathbb{R}^{2}\right)$ for any element $K \in \mathcal{T}_{h}$, the second term on the right-hand side of the above equation vanishes, namely,

$$
\lambda \sum_{K \in \mathcal{T}_{h}}\left(\Pi_{K}^{0} u\left(\mathbf{x}-\mathbf{M}_{K}\right), \nabla_{h} u_{\mathrm{CR}}^{\lambda \Pi_{h}^{0} u}-\nabla_{h} u_{\mathrm{CR}}\right)_{0, K}=0 .
$$

The third term on the right-hand side of (3.50) can by bounded by the superconvergence result of the RT element in Lemma 2.1 and the superclose property (3.2) of the CR element, which reads

$$
\left|\left(\sigma_{\mathrm{RT}}^{\lambda u}-\Pi_{\mathrm{RT}} \nabla u, \nabla_{h} u_{\mathrm{CR}}^{\lambda \Pi_{h}^{0} u}-\nabla_{h} u_{\mathrm{CR}}\right)\right| \lesssim h^{4}|\ln h|^{\frac{1}{2}}|u|_{\frac{7}{2}, \Omega}^{2} .
$$

It remains to analyze the first term on the right-hand side of (3.50). To this end, let $s_{h}=\Pi_{\mathrm{CR}} u-u_{\mathrm{CR}}^{\lambda \Pi_{h}^{0} u}$. Then, the commuting property of $\Pi_{\mathrm{CR}}$ gives

$$
\left(\nabla_{h}\left(u-u_{\mathrm{CR}}^{\lambda \Pi_{h}^{0} u}\right), \nabla_{h} u_{\mathrm{CR}}^{\lambda \Pi_{h}^{0} u}-\nabla_{h} u_{\mathrm{CR}}\right)=\left(\nabla_{h} s_{h}, \nabla_{h} u_{\mathrm{CR}}^{\lambda \Pi_{h}^{0} u}-\nabla_{h} u_{\mathrm{CR}}\right)
$$

It follows from the discrete eigenvalue problem (2.4) and the discrete source problem (2.18) that

$$
\left(\nabla_{h} s_{h}, \nabla_{h} u_{\mathrm{CR}}^{\lambda \Pi_{h}^{0} u}-\nabla_{h} u_{\mathrm{CR}}\right)=\left(s_{h}, \lambda \Pi_{h}^{0} u-\lambda_{\mathrm{CR}} u_{\mathrm{CR}}\right) .
$$

The error estimate (2.7) of the CR element implies

$$
\left\|\Pi_{\mathrm{CR}} u-u_{\mathrm{CR}}^{\lambda \Pi_{h}^{0} u}\right\|_{0, \Omega}+\left\|\Pi_{h}^{0}\left(u-u_{\mathrm{CR}}\right)\right\|_{0, \Omega}+\left|\lambda-\lambda_{\mathrm{CR}}\right| \lesssim h^{2}|u|_{2, \Omega} .
$$

Since

$$
\lambda \Pi_{h}^{0} u-\lambda_{\mathrm{CR}} u_{\mathrm{CR}}=\lambda \Pi_{h}^{0}\left(u-u_{\mathrm{CR}}\right)+\left(\lambda-\lambda_{\mathrm{CR}}\right) \Pi_{h}^{0} u_{\mathrm{CR}}+\lambda_{\mathrm{CR}}\left(\Pi_{h}^{0}-I\right) u_{\mathrm{CR}},
$$

a combination of (3.48), (3.51) and (3.52) leads to

$$
\left(\nabla_{h} s_{h}, \nabla_{h} u_{\mathrm{CR}}^{\lambda \Pi_{h}^{0} u}-\nabla_{h} u_{\mathrm{CR}}\right)=\lambda_{\mathrm{CR}}\left(s_{h},\left(\Pi_{h}^{0}-I\right) u_{\mathrm{CR}}\right)+O\left(h^{4}|u|_{2, \Omega}^{2}\right) .
$$

It follows from (3.50), the orthogonal property of the piecewise constant $L^{2}$ projection operator $\Pi_{h}^{0}$ and the above equation that

$$
I_{\mathrm{CR}}^{1}=-2 \lambda_{\mathrm{CR}}\left(\left(I-\Pi_{h}^{0}\right) s_{h},\left(I-\Pi_{h}^{0}\right) u_{\mathrm{CR}}\right)+O\left(h^{4}|\ln h|^{\frac{1}{2}}|u|_{\frac{7}{2}, \Omega}^{2}\right) .
$$

A substitution of Lemma 3.9 into the above identity completes the proof. 
3.6. Error estimate of $I_{\mathrm{CR}}^{2}=2\left(\Pi_{\mathrm{RT}} \nabla u-\sigma_{\mathrm{RT}}^{\lambda u} \sigma_{\mathrm{RT}}^{\lambda u}-\nabla_{h} u_{\mathrm{CR}}\right)$. The superconvergence of the RT element and the relation between the RT element and the CR element only lead to a suboptimal analysis of $I_{\mathrm{CR}}^{2}$. The key idea for an optimal analysis of $I_{\mathrm{CR}}^{2}$ is to exploit the $H\left(\right.$ div)-conformity and the divergence-free property of $\Pi_{\mathrm{RT}} \nabla u-\sigma_{\mathrm{RT}}^{\lambda u}$ in $I_{\mathrm{CR}}^{2}$.

Lemma 3.11. Suppose that $\left(\sigma_{\mathrm{RT}}^{\lambda u}, u_{\mathrm{RT}}^{\lambda u}\right)$ is the solution of source problem (2.13), $\left(\lambda_{\mathrm{CR}}, u_{\mathrm{CR}}\right)$ is the corresponding eigenpair of (2.4) by the $C R$ element and $u \in H^{\frac{7}{2}}(\Omega, \mathbb{R})$. Under the Assumption 2.2

$$
\left|I_{\mathrm{CR}}^{2}\right| \lesssim h^{4}|\ln h|^{\frac{1}{2}}|u|_{\frac{7}{2}, \Omega}^{2} .
$$

Proof. Thanks to the relation (2.20) between the CR element and the RT element, the term $I_{\mathrm{CR}}^{2}$ can be decomposed into the following two terms

$$
I_{\mathrm{CR}}^{2}=2\left(\Pi_{\mathrm{RT}} \nabla u-\sigma_{\mathrm{RT}}^{\lambda u}, \nabla_{h} u_{\mathrm{CR}}^{\lambda \Pi_{h}^{0} u}-\nabla_{h} u_{\mathrm{CR}}\right)-\lambda\left(\Pi_{\mathrm{RT}} \nabla u-\sigma_{\mathrm{RT}}^{\lambda u}, \Pi_{h}^{0} u\left(\mathbf{x}-\mathbf{M}_{K}\right)\right) .
$$

According to the superconvergence result in Lemma 2.1 and the superclose property (3.2) of the CR element,

$$
\left|\left(\Pi_{\mathrm{RT}} \nabla u-\sigma_{\mathrm{RT}}^{\lambda u}, \nabla_{h} u_{\mathrm{CR}}^{\lambda \Pi_{h}^{0} u}-\nabla_{h} u_{\mathrm{CR}}\right)\right| \lesssim h^{4}|\ln h|^{\frac{1}{2}}|u|_{\frac{7}{2}, \Omega}^{2} .
$$

To bound the second term on the right-hand side of (3.53), let $\tau_{h}=\Pi_{\mathrm{RT}} \nabla u-\sigma_{\mathrm{RT}}^{\lambda u}$ and $\phi_{K}=\frac{1}{2}\left(\left(x_{1}-M_{1}\right)^{2}+\left(x_{2}-M_{2}\right)^{2}\right)$ with the centroid $\mathbf{M}_{K}=\left(M_{1}, M_{2}\right)$. Since $\mathbf{x}-\mathbf{M}_{K}=\nabla \phi_{K}$ and $\operatorname{div} \tau_{h}=0$, it follows from the integration by parts and the continuity of $\tau_{h} \cdot \mathbf{n}$ that

$$
\left(\Pi_{\mathrm{RT}} \nabla u-\sigma_{\mathrm{RT}}^{\lambda u}, \Pi_{h}^{0} u\left(\mathbf{x}-\mathbf{M}_{K}\right)\right)=\sum_{e \in \mathcal{E}^{i}} \int_{e} \tau_{h} \cdot \mathbf{n}_{e}\left[\Pi_{K}^{0} u \phi_{K}\right] d s .
$$

Since $\phi_{K}=O\left(h^{2}\right)$, by the Cauchy-Schwarz inequality and the trace inequality,

$$
\left.\left|\int_{e}\left(\tau_{h} \cdot \mathbf{n}_{e}\right)\left[\Pi_{K}^{0} u \phi_{K}\right] d s\right| \lesssim h^{2}\left\|\tau_{h} \cdot \mathbf{n}_{e}\right\|_{0, e}\left\|\left[\Pi_{h}^{0} u\right]\right\|_{0, e} \lesssim h^{2}\left\|\tau_{h}\right\|_{0, \omega_{e}}|u|_{1, \omega_{e}}\right) .
$$

Consequently, thanks to the superconvergence result in Lemma 2.1 ,

$$
\left|\left(\Pi_{\mathrm{RT}} \nabla u-\sigma_{\mathrm{RT}}^{\lambda u}, \Pi_{h}^{0} u\left(\mathbf{x}-\mathbf{M}_{K}\right)\right)\right| \lesssim h^{2}\left\|\tau_{h}\right\|_{0, \Omega}\|u\|_{1, \Omega} \lesssim h^{4}|\ln h|^{\frac{1}{2}}|u|_{\frac{7}{2}, \Omega}^{2} .
$$

A substitution of (3.54) and (3.55) into (3.53) yields

$$
\left|I_{\mathrm{CR}}^{2}\right| \lesssim h^{4}|\ln h|^{\frac{1}{2}}|u|_{\frac{7}{2}, \Omega^{\prime}}^{2}
$$

which completes the proof.

The following asymptotic expansions of eigenvalues by the CR element come from the combination of Lemmas 3.5, 3.7, 3.8, 3.10, 3.11 and Theorem 3.1. 
Theorem 3.2. Suppose that $(\lambda, u)$ is the eigenpair of $(2.3)$ with $u \in H^{4}(\Omega, \mathbb{R}) \cap H_{0}^{1}(\Omega, \mathbb{R})$, and $\left(\lambda_{\mathrm{CR}}, u_{\mathrm{CR}}\right)$ is the corresponding eigenpair of (2.4) by the CR element on an uniform triangulation $\mathcal{T}_{h}$. It holds that

$$
\begin{aligned}
\lambda-\lambda_{\mathrm{CR}}= & h^{2}\left(\frac{\gamma_{\mathrm{RT}}^{11}}{4}\left\|\partial_{x_{1} x_{1}} u-\partial_{x_{2} x_{2}} u\right\|_{0, \Omega}^{2}+\gamma_{\mathrm{RT}}^{22}\left\|\partial_{x_{1} x_{2}} u\right\|_{0, \Omega}^{2}+\gamma_{\mathrm{RT}}^{12} \int_{\Omega}\left(\partial_{x_{1} x_{1}} u-\partial_{x_{2} x_{2}} u\right) \partial_{x_{1} x_{2}} u d x\right) \\
& -\frac{\lambda^{2}}{144} H^{2}+O\left(h^{4}|\ln h \| u|_{4, \Omega}^{2}\right) .
\end{aligned}
$$

3.7. Extrapolation eigenvalues. Denote the approximate eigenvalues of the CR element on $\mathcal{T}_{h}$ by $\lambda_{\mathrm{CR}}^{h}$. Suppose that eigenvalues of the CR element converge at a rate $\alpha$ with a fixed coefficient $C$, namely

$$
\lambda-\lambda_{\mathrm{CR}}^{h}=C h^{\alpha}+O\left(h^{\beta}\right) \text { with } \beta>\alpha .
$$

If the convergence rate $\alpha$ is known, define extrapolation eigenvalues

$$
\lambda_{\mathrm{CR}, 1}^{\mathrm{EXP}}=\frac{2^{\alpha} \lambda_{\mathrm{CR}}^{2 h}-\lambda_{\mathrm{CR}}^{h}}{2^{\alpha}-1} .
$$

It is easy to verify that extrapolation eigenvalues $\lambda_{\mathrm{CR}, 1}^{\mathrm{EXP}}$ in (3.57) converge to eigenvalues at a higher rate $\beta$. If eigenfunctions are smooth enough, say $u \in H^{4}(\Omega, \mathbb{R})$, Theorem 3.2 indicates that on uniform traingulations,

$$
\left|\lambda-\lambda_{\mathrm{CR}, 1}^{\mathrm{EXP}}\right| \lesssim h^{4}|\ln h||u|_{4, \Omega}^{2} .
$$

The extrapolation eigenvalues in (3.57) converge at a higher rate 4.

If eigenfunctions are singular, the convergence rate $\alpha$ in (3.56) is unknown. Suppose that the higher order term $O\left(h^{\beta}\right)$ is zero,

$$
\lambda_{\mathrm{CR}}^{4 h}-\lambda=4^{\alpha} C h^{\alpha}, \quad \lambda_{\mathrm{CR}}^{2 h}-\lambda=2^{\alpha} C h^{\alpha}, \quad \lambda_{\mathrm{CR}}^{h}-\lambda=C h^{\alpha} .
$$

Then,

$$
\frac{\lambda_{\mathrm{CR}}^{4 h}-\lambda_{\mathrm{CR}}^{2 h}}{\lambda_{\mathrm{CR}}^{2 h}-\lambda_{\mathrm{CR}}^{h}}=2^{\alpha}
$$

A substitution of the above relation into (3.57) gives new extrapolation eigenvalues

$$
\lambda_{\mathrm{CR}, 2}^{\mathrm{EXP}}=\frac{\left(\lambda_{\mathrm{CR}}^{4 h}-\lambda_{\mathrm{CR}}^{2 h}\right) \lambda_{\mathrm{CR}}^{h}-\left(\lambda_{\mathrm{CR}}^{2 h}-\lambda_{\mathrm{CR}}^{h}\right) \lambda_{\mathrm{CR}}^{2 h}}{\lambda_{\mathrm{CR}}^{4 h}+\lambda_{\mathrm{CR}}^{h}-2 \lambda_{\mathrm{CR}}^{2 h}}
$$

for unknown convergence rate $\alpha$ in (3.56).

\section{Asymptotic EXPANSIONS OF EIgENVALUES by the ECR ELEMENT}

Let $u_{\mathrm{ECR}}^{\lambda u} \in V_{\mathrm{ECR}}$ be the solution of the following discrete source problem

$$
\left(\nabla u_{\mathrm{ECR}}^{\lambda u}, \nabla_{h} v_{h}\right)=\left(\lambda u, v_{h}\right) \quad \text { for any } v_{h} \in V_{\mathrm{ECR}}
$$


The equivalence between the ECR element and the RT element [11] is crucial for expansions of eigenvalues by the ECR element. Thanks to (2.21) in Lemma 2.2, a similar proof to the one in Lemma 3.1 leads to

$$
\left\|\sigma_{\mathrm{RT}}^{\lambda u}-\nabla_{h} u_{\mathrm{ECR}}\right\|_{0, \Omega} \lesssim h^{2}|u|_{2, \Omega},
$$

provided that $u \in H^{2}(\Omega, \mathbb{R}) \cap H_{0}^{1}(\Omega, \mathbb{R})$.

Lemma 4.1. Suppose that $(\lambda, u)$ is the eigenpair of (2.3) with $u \in H^{\frac{7}{2}}(\Omega, \mathbb{R}) \cap H_{0}^{1}(\Omega, \mathbb{R})$, and $\left(\lambda_{\mathrm{ECR}}, u_{\mathrm{ECR}}\right)$ is the corresponding eigenpair of (2.4) by the ECR element. Under the Assumption 2.2 .

$$
\lambda-\lambda_{\mathrm{ECR}}=\left\|\nabla u-\Pi_{\mathrm{RT}} \nabla u\right\|_{0, \Omega}^{2}-2 \lambda\left(u-\Pi_{\mathrm{ECR}} u, u-\Pi_{h}^{0} u\right)+I_{\mathrm{ECR}}+O\left(h^{4}|\ln h \| u|_{\frac{7}{2}, \Omega}^{2}\right) .
$$

with

$$
I_{\mathrm{ECR}}=2\left(\nabla u-\Pi_{\mathrm{RT}} \nabla u, \sigma_{\mathrm{RT}}^{\lambda u}-\nabla_{h} u_{\mathrm{ECR}}\right) .
$$

Proof. A similar analysis to that for (3.12) in Theorem 3.1 leads to

$$
\lambda-\lambda_{\mathrm{ECR}}=\left\|\nabla_{h}\left(u-u_{\mathrm{ECR}}\right)\right\|_{0, \Omega}^{2}-2 \lambda\left(u-\Pi_{\mathrm{ECR}} u, u\right)+O\left(h^{4}|u|_{2, \Omega}^{2}\right) .
$$

With the interpolation $\Pi_{\mathrm{RT}} \nabla u$ and the solution $\sigma_{\mathrm{RT}}^{\lambda u}$ of the discrete source problem (2.13), the first term on the right-hand side of (4.4) can be decomposed as

$$
\begin{aligned}
\left\|\nabla u-\nabla_{h} u_{\mathrm{ECR}}\right\|_{0, \Omega}^{2}= & \left\|\nabla u-\Pi_{\mathrm{RT}} \nabla u\right\|_{0, \Omega}^{2}+\left\|\Pi_{\mathrm{RT}} \nabla u-\sigma_{\mathrm{RT}}^{\lambda u}\right\|_{0, \Omega}^{2}+\left\|\sigma_{\mathrm{RT}}^{\lambda u}-\nabla_{h} u_{\mathrm{ECR}}\right\|_{0, \Omega}^{2} \\
& +2\left(\Pi_{\mathrm{RT}} \nabla u-\sigma_{\mathrm{RT}}^{\lambda u} \sigma_{\mathrm{RT}}^{\lambda u}-\nabla_{h} u_{\mathrm{ECR}}\right)+I_{\mathrm{RT}}+I_{\mathrm{ECR},}
\end{aligned}
$$

with $I_{\mathrm{RT}}=2\left(\nabla u-\Pi_{\mathrm{RT}} \nabla u, \Pi_{\mathrm{RT}} \nabla u-\sigma_{\mathrm{RT}}^{\lambda u}\right)$ defined in (3.9). Since $\sigma_{\mathrm{RT}}^{\lambda u}$ is the RT element approximation of $\nabla u$, a combination of the superconvergence (2.15) of the RT element, (4.2) and the triangle inequality bounds the fourth term on the right-hand side of (4.5) as follows

$$
2\left|\left(\Pi_{\mathrm{RT}} \nabla u-\sigma_{\mathrm{RT}}^{\lambda u}, \sigma_{\mathrm{RT}}^{\lambda u}-\nabla_{h} u_{\mathrm{ECR}}\right)\right| \lesssim h^{4}|\ln h|^{1 / 2}|u|_{\frac{7}{2}, \Omega}|u|_{2, \Omega} .
$$

Since the second term on the right-hand side of (4.5) is anlyzed in the superconvergence result (2.15) of the RT element and the fifth term $I_{\mathrm{RT}}$ is estimated in Lemma 3.8, this and (4.2) give

$$
\left\|\nabla u-\nabla_{h} u_{\mathrm{ECR}}\right\|_{0, \Omega}^{2}=\left\|\nabla u-\Pi_{\mathrm{RT}} \nabla u\right\|_{0, \Omega}^{2}+I_{\mathrm{ECR}}+O\left(h^{4}|\ln h \| u|_{\frac{7}{2}, \Omega}^{2}\right) .
$$

By the definition of the interpolation $\Pi_{\mathrm{ECR}}$ in (2.6),

$$
\left(u-\Pi_{\mathrm{ECR}} u, u\right)=\left(u-\Pi_{\mathrm{ECR}} u, u-\Pi_{h}^{0} u\right) .
$$

A substitution of these two equations into (4.4) leads to

$$
\lambda-\lambda_{\mathrm{ECR}}=\left\|\nabla u-\Pi_{\mathrm{RT}} \nabla u\right\|_{0, \Omega}^{2}-2 \lambda\left(u-\Pi_{\mathrm{ECR}} u, u-\Pi_{h}^{0} u\right)+I_{\mathrm{ECR}}+O\left(h^{4}|\ln h \| u|_{\frac{7}{2}, \Omega}^{2}\right),
$$

which completes the proof.

For the ECR element, the following lemma analyzes a similar result to that in Lemma 3.6 for the CR element, namely, the superconvergence of the inner product of the canonical interpolations of the ECR element and the piecewise constant $L^{2}$ projection. 
Lemma 4.1. If two adjacent elements $K_{1}$ and $K_{2}$ form a parallelogram. For any $w \in P_{2}\left(K_{1} \cup K_{2}, \mathbb{R}\right)$ and $v \in P_{1}\left(K_{1} \cup K_{2}, \mathbb{R}\right)$ that

$$
\left(w-\Pi_{\mathrm{ECR}} w, v-\Pi_{h}^{0} v\right)_{0, K_{1} \cup K_{2}}=0 .
$$

Furthermore, under the Assumption 2.2 .

$$
\left|\left(\left(I-\Pi_{\mathrm{ECR}}\right) u,\left(I-\Pi_{h}^{0}\right) u\right)\right| \lesssim h^{4}|u|_{3, \Omega}^{2}
$$

provided that $u \in H^{3}(\Omega, \mathbb{R})$.

Proof. The main idea is to derive a refined expansion of the error $\Pi_{\mathrm{CR}} w-\Pi_{\mathrm{ECR}} w$ in terms of the second order tangential derivatives $\frac{\partial^{2} w}{\partial \mathbf{t}_{j}^{2}}$, where $\mathbf{t}_{j}$ are the tangential vectors of the three edges $e_{j}, j=1,2,3$, of element $K$. To this end, define $\phi_{\mathrm{ECR}} \in V_{\mathrm{ECR}}$ by

$$
\phi_{\mathrm{ECR}}(\mathbf{x})=2-\frac{36}{H^{2}} \sum_{i=1}^{2}\left(x_{i}-M_{i}\right)^{2}
$$

with the centroid $\mathbf{M}_{K}=\left(M_{1}, M_{2}\right)$ and $H^{2}=\sum_{i=1}^{3}\left|e_{i}\right|^{2}$. It is easy to verify that

$$
\int_{e_{i}} \phi_{\mathrm{ECR}} d s=0, \forall 1 \leq i \leq 3 \quad \text { and } \quad \frac{1}{|K|} \int_{K} \phi_{\mathrm{ECR}} d x=1 .
$$

Note that the quadratic function $\phi_{\mathrm{CR}}^{i}$ from Lemma 3.6 satisfies $\frac{1}{|K|} \int_{K} \phi_{\mathrm{CR}}^{i} d x=\frac{1}{9}$, and the integral average of $\phi_{\mathrm{CR}}^{i}$ on any edge is zero. Thus,

$$
\Pi_{\mathrm{ECR}} \phi_{\mathrm{CR}}^{i}=\frac{1}{9} \phi_{\mathrm{ECR}}
$$

It follows from the expansion of the interpolation error of the CR element in (3.36) that $w=\Pi_{\mathrm{CR}} w-\sum_{i=1}^{3} \frac{\left|e_{i}\right|^{2}}{8|K|} \int_{K} \frac{\partial^{2} w}{\partial t_{i}^{2}} d x \phi_{\mathrm{CR}}^{i}$. Thus,

$$
\Pi_{\mathrm{ECR}} w=\Pi_{\mathrm{CR}} w-\frac{1}{9} \phi_{\mathrm{ECR}} \sum_{i=1}^{3} \frac{\left|e_{i}\right|^{2}}{8|K|} \int_{K} \frac{\partial^{2} w}{\partial \mathbf{t}_{i}^{2}} d x .
$$

By the property of $\phi_{\mathrm{ECR}}$ in (4.8), $\phi_{\mathrm{ECR}}=\left(I-\Pi_{\mathrm{CR}}\right) \phi_{\mathrm{ECR}}$. This and the above identity yield

$$
\Pi_{\mathrm{CR}} w-\Pi_{\mathrm{ECR}} w=\frac{1}{9}\left(I-\Pi_{\mathrm{CR}}\right) \phi_{\mathrm{ECR}} \sum_{i=1}^{3} \frac{\left|e_{i}\right|^{2}}{8|K|} \int_{K} \frac{\partial^{2} w}{\partial \mathbf{t}_{i}^{2}} d x
$$

By the expansion of the inner product of the errors of the canonical interpolation of the CR element and the piecewise constant $L^{2}$ projection from (3.39), this leads to,

$$
\begin{aligned}
\left(\Pi_{\mathrm{CR}} w-\Pi_{\mathrm{ECR}} w, v-\Pi_{K}^{0} v\right)_{0, K} & =\frac{1}{9}\left(\left(I-\Pi_{\mathrm{CR}}\right) \phi_{\mathrm{ECR},} v-\Pi_{K}^{0} v\right)_{0, K} \sum_{i=1}^{3} \frac{\left|e_{i}\right|^{2}}{8|K|} \int_{K} \frac{\partial^{2} w}{\partial \mathbf{t}_{i}^{2}} d x \\
& =-\frac{1}{2430} \sum_{j=1}^{3} \frac{\partial^{2} \phi_{\mathrm{ECR}}}{\partial \mathbf{t}_{j}^{2}}\left|e_{j}\right|^{2}|K| \nabla v \cdot \overrightarrow{\boldsymbol{m}_{j} \boldsymbol{p}_{j}} \sum_{i=1}^{3} \frac{\left|e_{i}\right|^{2}}{8|K|} \int_{K} \frac{\partial^{2} w}{\partial \mathbf{t}_{i}^{2}} d x
\end{aligned}
$$


A direct calculation derives $\frac{\partial^{2} \phi_{\mathrm{ECR}}}{\partial \mathrm{t}_{j}^{2}}=-\frac{72}{H^{2}}, \forall 1 \leq j \leq 3$. Thus,

$$
\left(\Pi_{\mathrm{CR}} w-\Pi_{\mathrm{ECR}} w, v-\Pi_{h}^{0} v\right)_{0, K}=\frac{4|K|}{135 H^{2}}\left(\sum_{i=1}^{3} \frac{\left|e_{i}\right|^{2}}{8|K|} \int_{K} \frac{\partial^{2} w}{\partial \mathbf{t}_{i}^{2}} d x\right) \sum_{j=1}^{3}\left|e_{j}\right|^{2} \nabla v \cdot{\overrightarrow{\boldsymbol{m}_{j} \boldsymbol{p}_{j}}}_{.}
$$

For any $w \in P_{2}\left(K_{1} \cup K_{2}, \mathbb{R}\right)$ and $v \in P_{1}\left(K_{1} \cup K_{2}, \mathbb{R}\right)$, Assumption 2.2 implies that the term $\frac{|K|}{H^{2}}\left(\sum_{i=1}^{3} \frac{\left|e_{i}\right|^{2}}{8|K|} \int_{K} \frac{\partial^{2} w}{\partial t_{i}^{2}} d x\right)$ has the same value on elements $K_{1}$ and $K_{2}$, thus,

$$
\left(\Pi_{\mathrm{CR}} w-\Pi_{\mathrm{ECR}} w, v-\Pi_{h}^{0} v\right)_{0, K_{1} \cup K_{2}}=0 .
$$

A combination of Lemma 3.6 for the CR element and (4.9) leads to

$$
\left(w-\Pi_{\mathrm{ECR}} w, v-\Pi_{h}^{0} v\right)_{0, K_{1} \cup K_{2}}=0,
$$

which completes the proof for (4.6).

It follows from a similar proof for Lemma 3.6 and (4.6) that

$$
\left|\left(\left(I-\Pi_{\mathrm{ECR}}\right) u, u-\Pi_{h}^{0} u\right)\right| \lesssim h^{4}|u|_{3, \Omega}^{2},
$$

which completes the proof.

It remains to analyze the term $I_{\mathrm{ECR}}$. Thanks to the superconvergence result (2.15) for $\Pi_{\mathrm{RT}} \nabla u-\sigma_{\mathrm{RT}}^{\lambda u}$, and the equivalence (2.21) between the ECR element and the RT element, namely $\sigma_{\mathrm{RT}}^{\lambda u}=\nabla_{h} u_{\mathrm{ECR}}^{\lambda \Pi_{h}^{0} u}$,

$I_{\mathrm{ECR}}=2\left(\nabla u-\Pi_{\mathrm{RT}} \nabla u, \sigma_{\mathrm{RT}}^{\lambda u}-\nabla_{h} u_{\mathrm{ECR}}\right)=2\left(\nabla u-\nabla_{h} u_{\mathrm{ECR}}^{\lambda \Pi_{h}^{0} u}, \nabla_{h} u_{\mathrm{ECR}}^{\lambda \Pi_{h}^{0} u}-\nabla_{h} u_{\mathrm{ECR}}\right)+O\left(h^{4}|\ln h|^{\frac{1}{2}}|u|_{\frac{7}{2}, \Omega}^{2}\right)$.

A similar proof to that in Lemma 3.10 for a similar term $I_{\mathrm{CR}}^{1}$ of the CR element leads to

$$
I_{\mathrm{ECR}}=-2\left(\left(I-\Pi_{h}^{0}\right) s_{h}, \lambda_{\mathrm{ECR}}\left(I-\Pi_{h}^{0}\right) u_{\mathrm{ECR}}\right)+O\left(h^{4}|\ln h|^{\frac{1}{2}}|u|_{\frac{7}{2}, \Omega}^{2}\right),
$$

with $s_{h}=\Pi_{\mathrm{ECR}} u-u_{\mathrm{ECR}}^{\lambda \Pi_{h}^{0} u}$.

Lemma 4.2. Let $\left(\lambda_{\mathrm{ECR}}, u_{\mathrm{ECR}}\right)$ be the eigenpair of (2.4) by the ECR element and $u_{\mathrm{ECR}}^{\lambda \Pi_{h}^{0} u}$ be the solution of (2.19). Suppose $u \in H^{\frac{7}{2}}\left(\Omega, \mathbb{R}^{2}\right)$. Under the Assumption 2.2

$$
\left|I_{\mathrm{ECR}}\right| \lesssim h^{4}|\ln h|^{\frac{1}{2}}|u|_{\frac{7}{2}, \Omega}^{2} \text {. }
$$

Proof. It only needs to estimate the first term on the right-hand side of (4.10). Since both $s_{h}$ and $u_{\mathrm{ECR}}$ are piecewise quadratic polynomials, the analysis in Lemma 3.9 can not be directly employed. In fact, it follows from the orthogonal property of the piecewise constant $L^{2}$ projection that there is the following decomposition:

$$
\left(\left(I-\Pi_{h}^{0}\right) s_{h},\left(I-\Pi_{h}^{0}\right) u_{\mathrm{ECR}}\right)=\left(\left(I-\Pi_{h}^{0}\right) s_{h},\left(I-\Pi_{\mathrm{CR}}\right) u_{\mathrm{ECR}}\right)+\left(\left(I-\Pi_{h}^{0}\right) s_{h},\left(I-\Pi_{h}^{0}\right) \Pi_{\mathrm{CR}} u_{\mathrm{ECR}}\right) .
$$

Since $\left\|\nabla_{h} s_{h}\right\|_{0, \Omega} \lesssim h|u|_{2, \Omega}$, the first term on the right-hand side of the above equation can be estimated as

$$
\left(\left(I-\Pi_{h}^{0}\right) s_{h},\left(I-\Pi_{\mathrm{CR}}\right) u_{\mathrm{ECR}}\right) \leq\left\|\left(I-\Pi_{h}^{0}\right) s_{h}\right\|_{0, \Omega}\left\|\left(I-\Pi_{\mathrm{CR}}\right) u_{\mathrm{ECR}}\right\| \lesssim h^{4}|u|_{2, \Omega}^{2}
$$


A substitution of this estimate into the above equation leads to

$$
\begin{aligned}
I_{\mathrm{ECR}}= & -2\left(\left(I-\Pi_{h}^{0}\right) s_{h}, \lambda_{\mathrm{ECR}}\left(I-\Pi_{h}^{0}\right) \Pi_{\mathrm{CR}} u_{\mathrm{ECR}}\right)+O\left(h^{4}|\ln h|^{\frac{1}{2}}|u|_{\frac{7}{2}, \Omega}^{2}\right) \\
= & -2\left(\left(I-\Pi_{h}^{0}\right)\left(I-\Pi_{\mathrm{CR}}\right) s_{h}, \lambda_{\mathrm{ECR}}\left(I-\Pi_{h}^{0}\right) \Pi_{\mathrm{CR}} u_{\mathrm{ECR}}\right) \\
& -2\left(\left(I-\Pi_{h}^{0}\right) \Pi_{\mathrm{CR} s_{h}}, \lambda_{\mathrm{ECR}}\left(I-\Pi_{h}^{0}\right) \Pi_{\mathrm{CR}} u_{\mathrm{ECR}}\right)+O\left(h^{4}|\ln h|^{\frac{1}{2}}|u|_{\frac{7}{2}, \Omega}^{2}\right) .
\end{aligned}
$$

A similar analysis to that in Lemma 3.10 for the CR element proves

$$
\left|-2\left(\left(I-\Pi_{h}^{0}\right) \Pi_{\mathrm{CR}} s_{h}, \lambda_{\mathrm{ECR}}\left(I-\Pi_{h}^{0}\right) \Pi_{\mathrm{CR}} u_{\mathrm{ECR}}\right)\right| \lesssim h^{4}|\ln h|^{\frac{1}{2}}|u|_{\frac{7}{2}, \Omega}^{2} .
$$

Thus,

$$
I_{\mathrm{ECR}}=-2\left(\left(I-\Pi_{h}^{0}\right)\left(I-\Pi_{\mathrm{CR}}\right) s_{h}, \lambda_{\mathrm{ECR}}\left(I-\Pi_{h}^{0}\right) \Pi_{\mathrm{CR}} u_{\mathrm{ECR}}\right)+O\left(h^{4}|\ln h|^{\frac{1}{2}}|u|_{\frac{7}{2}, \Omega}^{2}\right) .
$$

Given any element $K \in \mathcal{T}_{h}$, recall $\phi_{\mathrm{ECR}}(\mathbf{x})$ defined in (4.7):

$$
\phi_{\mathrm{ECR}}(\mathbf{x})=2-\frac{36}{H^{2}} \sum_{i=1}^{2}\left(x_{i}-M_{i}\right)^{2}
$$

with $\mathbf{M}_{K}=\left(M_{1}, M_{2}\right)$ the centroid of element $K$ and $H^{2}=\sum_{i=1}^{3}\left|e_{i}\right|^{2}$. Define

$$
\phi_{\mathrm{ECR}}^{1}(\mathbf{x})=\left(x_{1}-M_{1}\right)^{2}-\left(x_{2}-M_{2}\right)^{2}, \quad \phi_{\mathrm{ECR}}^{2}(\mathbf{x})=\left(x_{1}-M_{1}\right)\left(x_{2}-M_{2}\right) .
$$

Note that the functions $\phi_{\mathrm{ECR}}^{1}$, and $\phi_{\mathrm{ECR}}^{2}$ belong to the compliment space of the shape function space of the ECR element with respect to $P_{2}(K)$. Since $\int_{e_{i}} \phi_{\mathrm{ECR}} d s=0$ for edge $e_{i}$ of $K, i=1,2,3$,

$$
\left.\left(I-\Pi_{\mathrm{CR}}\right) s_{h}\right|_{K}=\alpha_{K} \phi_{\mathrm{ECR}}(\mathbf{x}) \text { with } \alpha_{K}=-\left.\frac{H^{2}}{144} \Delta s_{h}\right|_{K} .
$$

Since there exists the expansion $\left.\left(I-\Pi_{K}^{0}\right) \Pi_{\mathrm{CR}} u_{\mathrm{ECR}}\right|_{K}=\sum_{j=1}^{2}\left(x_{j}-M_{j}\right) \partial_{j} \Pi_{\mathrm{CR}} u_{\mathrm{ECR}}$ and $\int_{K}\left(x_{j}-\right.$ $\left.M_{j}\right) d x=0$, this leads to

$$
I_{\mathrm{ECR}}=-\frac{\lambda_{\mathrm{ECR}}}{2} \sum_{K \in \mathcal{T}_{h}} \sum_{i, j=1}^{2} \int_{K} \Delta s_{h} \partial_{j} \Pi_{\mathrm{CR}} u_{\mathrm{ECR}}\left(x_{i}-M_{i}\right)^{2}\left(x_{j}-M_{j}\right) d x+O\left(h^{4}|\ln h|^{\frac{1}{2}}|u|_{\frac{7}{2}, \Omega}^{2}\right) .
$$

Define $a_{i i j}^{K}=\frac{1}{|K|} \int_{K}\left(x_{i}-M_{i}\right)^{2}\left(x_{j}-M_{j}\right) d x$. Note that $a_{i i j}^{K}=O\left(h^{3}\right), \sum_{K \in \mathcal{T}_{h}}\left\|\Delta s_{h}\right\|_{0, K}^{2} \lesssim|u|_{2, \Omega}^{2}$, and $\left\|\nabla_{h}\left(u-\Pi_{\mathrm{CR}} u_{\mathrm{ECR}}\right)\right\|_{0, \Omega} \lesssim h|u|_{2, \Omega}$. This leads to

$$
I_{\mathrm{ECR}}=-\frac{\lambda_{\mathrm{ECR}}}{2} \sum_{K \in \mathcal{T}_{h}} \sum_{i, j=1}^{2} a_{i i j}^{K} \int_{K} \Delta s_{h} \partial_{j} u d x+O\left(h^{4}|\ln h|^{\frac{1}{2}}|u|_{\frac{7}{2}, \Omega}^{2}\right) .
$$

In addition, there holds that

$$
a_{i i j}^{K_{1}}=-a_{i i j}^{K_{2}}, i, j=1,2
$$


provided that $K_{1}$ and $K_{2}$ form a parallelogram. Recall that the partition $\mathcal{T}_{h}$ of domain $\Omega$ includes the set of parallelograms $\mathcal{N}_{1}$ and the set of a few remaining boundary triangles $\mathcal{N}_{2}$ with $\kappa=\left|\mathcal{N}_{2}\right|$ the number of the elements in $\mathcal{N}_{2}$. For a parallelogram $K_{1} \cup K_{2}=: Q \in \mathcal{N}_{1}$, let $a_{i i j}^{Q}=a_{i i j}^{K_{1}}=-a_{i i j}^{K_{2}}$. Then,

$$
\begin{aligned}
\sum_{K \in \mathcal{T}_{h}} \sum_{i, j=1}^{2} a_{i i j}^{K} \int_{K} \Delta s_{h} \partial_{j} u d x= & \sum_{Q \in \mathcal{N}_{1}} \sum_{i, j=1}^{2} a_{i i j}^{Q}\left(\int_{K_{1}} \Delta s_{h} \partial_{j} u d x-\int_{K_{2}} \Delta s_{h} \partial_{j} u d x\right) \\
& +\sum_{K \in \mathcal{N}_{2}} \sum_{i, j=1}^{2} a_{i i j}^{K} \int_{K} \Delta s_{h} \partial_{j} u d x .
\end{aligned}
$$

Since the second term on the right-hand side of the above equation can be bounded as follows

$$
\sum_{K \in \mathcal{N}_{2}} \int_{K} \Delta s_{h} \partial_{j} u d x \leq \sum_{K \in \mathcal{N}_{2}} \sqrt{|K|}\left\|\Delta s_{h}\right\|_{0, K}\left\|\partial_{j} u\right\|_{0, \infty} \lesssim \sqrt{\kappa} h\|u\|_{3, \Omega}^{2}
$$

it only needs to analyze the first term. To this end, introduce the $L^{2}$ projection operators $\Pi_{Q}^{\ell}: L^{2}(Q, \mathbb{R}) \rightarrow P_{\ell}(Q, \mathbb{R}), \ell=1,2$, for $w \in L^{2}(Q, \mathbb{R})$

$$
\int_{Q} \nabla^{i} \Pi_{Q}^{\ell} w d x=\int_{Q} \nabla^{i} w d x \quad \forall 0 \leq i \leq \ell
$$

This leads to the following decomposition:

$$
\begin{aligned}
& \int_{K_{1}} \Delta \Pi_{\mathrm{ECR}} u \partial_{j} u d x-\int_{K_{2}} \Delta \Pi_{\mathrm{ECR}} u \partial_{j} u d x \\
& =\int_{K_{1}} \Delta \Pi_{\mathrm{ECR}}\left(I-\Pi_{Q}^{2}\right) u \partial_{j}\left(I-\Pi_{Q}^{1}\right) u d x+\int_{K_{1}} \Delta \Pi_{\mathrm{ECR}}\left(I-\Pi_{Q}^{2}\right) u \partial_{j} \Pi_{Q}^{1} u d x \\
& \quad+\int_{K_{1}} \Delta \Pi_{Q}^{2} u \partial_{j}\left(I-\Pi_{Q}^{1}\right) u d x-\left(\int_{K_{2}} \Delta \Pi_{\mathrm{ECR}}\left(I-\Pi_{Q}^{2}\right) u \partial_{j}\left(I-\Pi_{Q}^{1}\right) u d x\right. \\
& \left.\quad+\int_{K_{2}} \Delta \Pi_{\mathrm{ECR}}\left(I-\Pi_{Q}^{2}\right) u \partial_{j} \Pi_{Q}^{1} u d x+\int_{K_{2}} \Delta \Pi_{Q}^{2} u \partial_{j}\left(I-\Pi_{Q}^{1}\right) u d x\right) \\
& \quad+\int_{K_{1}} \Delta \Pi_{\mathrm{ECR}} \Pi_{Q}^{2} u \partial_{j} \Pi_{Q}^{1} u d x-\int_{K_{2}} \Delta \Pi_{\mathrm{ECR}} \Pi_{Q}^{2} u \partial_{j} \Pi_{Q}^{1} u d x
\end{aligned}
$$

The first eight terms on the right-hand side of the above equation can be bounded by $O(h)|u|_{3, Q}^{2}$. In order to analyze the last two terms, let $w=\Pi_{Q}^{2} u$. Then, for $K=K_{1}, K_{2}$, it holds that

$$
\left(I-\Pi_{\mathrm{ECR}}\right) w=\frac{\left\|\partial_{x_{1} x_{1}} w-\partial_{x_{2} x_{2}} w\right\|_{0, K}}{4|K|^{\frac{1}{2}}}\left(I-\Pi_{\mathrm{ECR}}\right) \phi_{\mathrm{ECR}}^{1}+\frac{\left\|\partial_{x_{1} x_{2}} w\right\|_{0, K}}{|K|^{\frac{1}{2}}}\left(I-\Pi_{\mathrm{ECR}}\right) \phi_{\mathrm{ECR}}^{2}
$$


with $\phi_{\mathrm{ECR}}^{i}, i=1,2$, defined in (4.11). Apply the Laplacian operator to both sides of the above equation,

$$
\Delta \Pi_{\mathrm{ECR}} w=\Delta w+\frac{\left\|\partial_{x_{1} x_{1}} w-\partial_{x_{2} x_{2}} w\right\|_{0, K}}{4|K|^{\frac{1}{2}}} \Delta\left(\Pi_{\mathrm{ECR}} \phi_{\mathrm{ECR}}^{1}\right)+\frac{\left\|\partial_{x_{1} x_{2}} w\right\|_{0, K}}{|K|^{\frac{1}{2}}} \Delta\left(\Pi_{\mathrm{ECR}} \phi_{\mathrm{ECR}}^{2}\right) .
$$

Note that on both $K=K_{1}$ and $K=K_{2}, \Delta\left(\phi_{\mathrm{ECR}}^{i}\right)_{K}=0, i=1,2$. Here $(\cdot)_{K}$ denotes the corresponding function for element $K$. In addition, a similar relation holds for the interpolation of the ECR element, namely, $\Delta\left(\Pi_{\mathrm{ECR}} \phi_{\mathrm{ECR}}^{i}\right)_{K_{1}}=\Delta\left(\Pi_{\mathrm{ECR}} \phi_{\mathrm{ECR}}^{i}\right)_{K_{2}}, i=1,2$. As a result, the last two terms on the right-hand side of (4.12) cancel each other, and

$$
\left|\int_{K_{1}} \Delta \Pi_{\mathrm{ECR}} u \partial_{j} u d x-\int_{K_{2}} \Delta \Pi_{\mathrm{ECR}} u \partial_{j} u d x\right| \lesssim h|u|_{3, Q}^{2} .
$$

A similar argument gives

$$
\left|\int_{K_{1}}\left(\Pi_{K_{1}}^{0} \Delta u\right) \partial_{j} u d x-\int_{K_{2}}\left(\Pi_{K_{2}}^{0} \Delta u\right) \partial_{j} u d x\right| \lesssim h|u|_{3, Q}^{2} .
$$

A combination of (2.13), (2.21) and $-\Delta u=\lambda u$ leads to

$$
\Delta s_{h}=\Delta \Pi_{\mathrm{ECR}} u-\operatorname{div} \sigma_{\mathrm{RT}}^{\lambda u}=\Delta \Pi_{\mathrm{ECR}} u-\Pi_{h}^{0} \Delta u .
$$

Thus, a summary of these equations completes the proof.

In the following theorem, asymptotic expansions of eigenvalues of the ECR element are established and employed to improve the accuracy of approximate eigenvalues from second order to forth order by extrapolation methods.

Extrapolation eigenvalues of the ECR element follow from the similar definitions in (3.57) and (3.58). Denote the approximate eigenvalues of the ECR element on $\mathcal{T}_{h}$ by $\lambda_{\mathrm{ECR}}^{h}$. If eigenfunctions are smooth enough, define extrapolation eigenvalues by

$$
\lambda_{\mathrm{ECR}, 1}^{\mathrm{EXP}}=\frac{2^{\alpha} \lambda_{\mathrm{ECR}}^{2 h}-\lambda_{\mathrm{ECR}}^{h}}{2^{\alpha}-1}
$$

with $\alpha=2$. If eigenfunctions are singular, define

$$
\lambda_{\mathrm{ECR}, 2}^{\mathrm{EXP}}=\frac{\left(\lambda_{\mathrm{ECR}}^{4 h}-\lambda_{\mathrm{ECR}}^{2 h}\right) \lambda_{\mathrm{ECR}}^{h}-\left(\lambda_{\mathrm{ECR}}^{2 h}-\lambda_{\mathrm{ECR}}^{h}\right) \lambda_{\mathrm{ECR}}^{2 h} .}{\lambda_{\mathrm{ECR}}^{4 h}+\lambda_{\mathrm{ECR}}^{h}-2 \lambda_{\mathrm{ECR}}^{2 h}} .
$$

Theorem 4.1. Suppose that $(\lambda, u)$ is the eigenpair of $(2.3)$ with $u \in H^{4}(\Omega, \mathbb{R}) \cap H_{0}^{1}(\Omega, \mathbb{R})$, and $\left(\lambda_{\mathrm{ECR}}, u_{\mathrm{ECR}}\right)$ is the corresponding eigenpair of (2.4) by the ECR element. Under the Assumption 2.2

$$
\begin{aligned}
\lambda-\lambda_{\mathrm{ECR}}= & h^{2}\left(\frac{\gamma_{\mathrm{RT}}^{11}}{4}\left\|\partial_{x_{1} x_{1}} u-\partial_{x_{2} x_{2}} u\right\|_{0, \Omega}^{2}+\gamma_{\mathrm{RT}}^{12} \int_{\Omega}\left(\partial_{x_{1} x_{1}} u-\partial_{x_{2} x_{2}} u\right) \partial_{x_{1} x_{2}} u d x\right. \\
& \left.+\gamma_{\mathrm{RT}}^{22}\left\|\partial_{x_{1} x_{2}} u\right\|_{0, \Omega}^{2}\right)+O\left(h^{4}|\ln h \| u|_{4, \Omega}^{2}\right)
\end{aligned}
$$

with constants $\left\{\gamma_{\mathrm{RT}}^{i j}\right\}_{i, j=1}^{2}$ defined in (3.16). 
Therefore, extrapolation eigenvalues converge at a higher rate 4, namely,

$$
\left|\lambda_{\mathrm{ECR}, 1}^{\mathrm{EXP}}-\lambda\right| \lesssim h^{4}|\ln h \| u|_{4, \Omega}^{2} .
$$

\section{Numerical EXAMPLES}

This section presents three numerical tests. The first example computes eigenvalues of the Laplacian operator on a unit square, the second one deals with eigenvalues of a second order elliptic operator with discontinuous coefficients, and the last one computes eigenvalues on a cracked domain.

5.1. Example 1. In this example, the model problem (2.3) on the unit square $\Omega=(0,1)^{2}$ is considered. The exact eigenvalues are

$$
\lambda=\left(m^{2}+n^{2}\right) \pi^{2}, m, n \text { are positive integers, }
$$

and the corresponding eigenfunctions are $u=2 \sin \left(m \pi x_{1}\right) \sin \left(n \pi x_{2}\right)$. Since these eigenfunctions are smooth, the convergence rates of the eigenvalues are known to be $\alpha=2$. According to Theorem 3.2 and 4.1 , the extrapolation eigenvalues $\lambda_{\mathrm{CR}, 1}^{\mathrm{EXP}}$ in (3.57) and $\lambda_{\mathrm{ECR}, 1}^{\mathrm{EXP}}$ in (4.13) converge at same rate 4 on uniform triangulations.

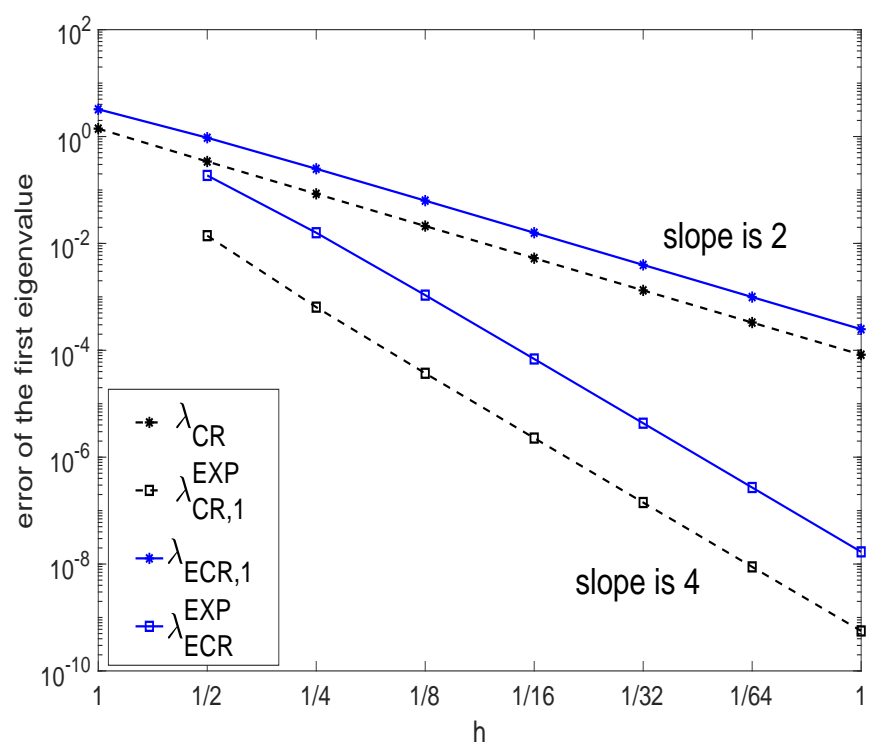

FIGURE 2. The errors of the extrapolation eigenvalues on an uniform triangulation for Example 1.

Figure 2 plots the errors of the first approximate eigenvalues of the CR element, the ECR element and their corresponding extrapolation eigenvalues on uniform triangulations. The initial triangulation $\mathcal{T}_{1}$ consists of two right triangles, obtained by cutting the unit square with a north-east line. Each triangulation $\mathcal{T}_{i}$ is refined into a half-sized triangulation uniformly, to get a higher level triangulation $\mathcal{T}_{i+1}$. Figure 2 verifies the optimal 
convergence rate 4 of the extrapolation eigenvalues $\lambda_{\mathrm{CR}, 1}^{\mathrm{EXP}}$ and $\lambda_{\mathrm{ECR}, 1}^{\mathrm{EXP}}$ in Theorem 3.2 and 4.1, respectively.

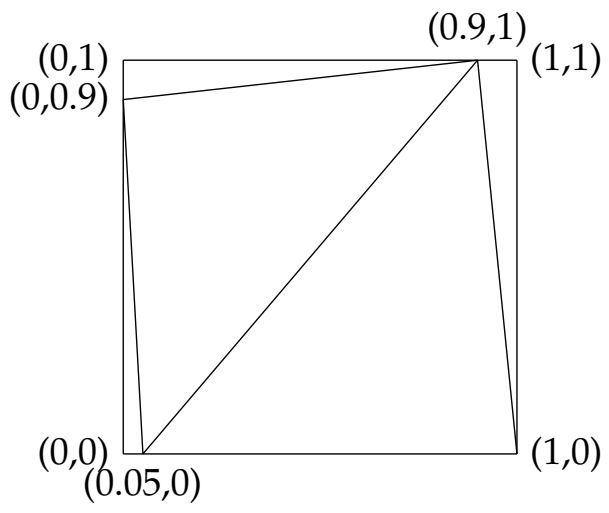

FIGURE 3. A level one triangulation $\mathcal{T}_{1}$ of $\Omega$.

Consider the eigenvalue problem on other triangulations with the initial triangulation $\mathcal{T}_{1}$ in Figure 3, Each triangulation $\mathcal{T}_{i}$ is refined into a half-sized triangulation uniformly to get a higher level triangulation $\mathcal{T}_{i+1}$. The errors of the corresponding eigenvalues $\lambda_{\mathrm{CR}}$, $\lambda_{\mathrm{ECR}}, \lambda_{\mathrm{CR}, 1}^{\mathrm{EXP}}$ and $\lambda_{\mathrm{ECR}, 1}^{\mathrm{EXP}}$ are recorded in Table1. Although these triangulations do not satisfy Assumption 2.2 anymore, most adjacent triangles form a nearly parallelogram. The percentage of this kind of triangles in $\mathcal{T}_{i}$ increases as the level $i$ grows. Note that the superconvergence of the extrapolation eigenvalues comes from the fact that some components of $\left\|\sigma_{\mathrm{RT}}^{\lambda u}-\Pi_{\mathrm{RT}} \nabla u\right\|_{0, \Omega}$ get canceled within nearly parallelograms. As the percentage of adjacent triangles forming nearly parallelograms increases, the accuracy of the extrapolation eigenvalues increases and tends to the one on uniform triangulations. Table 1 shows that on such triangulations, which are not uniform anymore, the convergence rates of the extrapolation eigenvalues are still over 3.

5.2. Example 2. This experiment considers the following eigenvalue problem

$$
\left\{\begin{aligned}
-\operatorname{div}(A \nabla u) & =\lambda u & & \text { in } \Omega, \\
u & =0 & & \text { on } \Gamma_{1} \cup \Gamma_{2}, \\
\frac{\partial u}{\partial n} & =0 & & \text { on } \Gamma_{3},
\end{aligned}\right.
$$

where $A=2$ if $x_{2}<1$ and $A=1$ if $x_{2}>1$. The domain is

$$
\Omega=\left\{\left(x_{1}, x_{2}\right) \in \mathbb{R}^{2}: 0<x_{2}<\sqrt{3} x_{1}, \sqrt{3}\left(1-x_{1}\right)<x_{2}\right\}
$$




\begin{tabular}{c|ccccccc}
\hline & $\mathcal{T}_{2}$ & $\mathcal{T}_{3}$ & $\mathcal{T}_{4}$ & $\mathcal{T}_{5}$ & $\mathcal{T}_{6}$ & $\mathcal{T}_{7}$ & $\mathcal{T}_{8}$ \\
\hline$\left|\lambda-\lambda_{\mathrm{CR}}\right|$ & 0.928068 & $2.22 \mathrm{E}-01$ & $5.55 \mathrm{E}-02$ & $1.39 \mathrm{E}-02$ & $3.48 \mathrm{E}-03$ & $8.69 \mathrm{E}-04$ & $2.17 \mathrm{E}-04$ \\
rate & & 2.07 & 2.00 & 2.00 & 2.00 & 2.00 & 2.00 \\
\hline$\left|\lambda-\lambda_{\mathrm{CR}, 1}^{\mathrm{EXP}}\right|$ & 2.870925 & $1.39 \mathrm{E}-02$ & $7.97 \mathrm{E}-05$ & $3.45 \mathrm{E}-05$ & $3.55 \mathrm{E}-06$ & $3.04 \mathrm{E}-07$ & $2.39 \mathrm{E}-08$ \\
rate & & 4.83 & 7.45 & 1.21 & 3.28 & 3.55 & 3.67 \\
\hline$\left|\lambda-\lambda_{\mathrm{ECR}}\right|$ & 2.683924 & $7.68 \mathrm{E}-01$ & $2.01 \mathrm{E}-01$ & $5.07 \mathrm{E}-02$ & $1.27 \mathrm{E}-02$ & $3.18 \mathrm{E}-03$ & $7.96 \mathrm{E}-04$ \\
rate & & 1.80 & 1.94 & 1.98 & 2.00 & 2.00 & 2.00 \\
\hline$\left|\lambda-\lambda_{\mathrm{ECR}, 1}^{\mathrm{EXP}}\right|$ & 2.825196 & $1.30 \mathrm{E}-01$ & $1.12 \mathrm{E}-02$ & $7.78 \mathrm{E}-04$ & $5.08 \mathrm{E}-05$ & $3.27 \mathrm{E}-06$ & $2.09 \mathrm{E}-07$ \\
rate & & 4.44 & 3.53 & 3.85 & 3.94 & 3.96 & 3.96 \\
\hline
\end{tabular}

TABLE 1. The errors of the extrapolation eigenvalues on nonuniform triangulations for Example 1.

with boundaries

$$
\begin{aligned}
& \Gamma_{1}=\left\{\left(x_{1}, x_{2}\right) \in \mathbb{R}^{2}: x_{2}=\sqrt{3} x_{1}, 0.5 \leq x_{1} \leq 1\right\}, \\
& \Gamma_{2}=\left\{\left(x_{1}, x_{2}\right) \in \mathbb{R}^{2}: x_{2}=\sqrt{3}\left(1-x_{1}\right), 0.5 \leq x_{1} \leq 1\right\}, \\
& \Gamma_{3}=\left\{\left(x_{1}, x_{2}\right) \in \mathbb{R}^{2}: x_{1}=1,0 \leq x_{2} \leq \sqrt{3}\right\} .
\end{aligned}
$$

The level one triangulation $\mathcal{T}_{1}$ is obtained by refining the domain $\Omega$ into four half-sized triangles. Each triangulation $\mathcal{T}_{i}$ is refined into a half-sized triangulation uniformly, to get a higher level triangulation $\mathcal{T}_{i+1}$. Since the exact eigenvalues of this problem are unknown, we take the first eigenvalue by the conforming $\mathrm{P}_{3}$ element on the mesh $\mathcal{T}_{9}$ as the reference eigenvalue.

Figure 4 presents the errors of the first approximate eigenvalues of the CR element, the ECR element and their corresponding extrapolation eigenvalues on the uniform triangulations. As showed in Figure 4, the eigenvalues $\lambda_{\mathrm{CR}}$ and $\lambda_{\mathrm{ECR}}$ converge at the rate 2 and the extrapolation eigenvalues $\lambda_{\mathrm{CR}, 1}^{\mathrm{EXP}}, \lambda_{\mathrm{CR}, 2}^{\mathrm{EXP}}, \lambda_{\mathrm{ECR}, 1}^{\mathrm{EXP}}$ and $\lambda_{\mathrm{ECR}, 2}^{\mathrm{EX}}$ converge at the same rate 4. This confirms the theoretical results in Theorem 3.2 and 4.1. Note that the errors of the extrapolation eigenvalues $\lambda_{\mathrm{CR}, 2}^{\mathrm{EXP}}$ and $\lambda_{\mathrm{ECR}, 2}^{\mathrm{EXP}}$ are slightly less than $\lambda_{\mathrm{CR}, 1}^{\mathrm{EXP}}$ and $\lambda_{\mathrm{ECR}, 1}^{\mathrm{EXP}}$ respectively.

5.3. Example 3. This example considers the model problem (2.3) on a crack domain $\Omega$ (see Figure 5). A Dirichlet condition is applied on the boundary $\Gamma_{D}$ of the non-cracked domain $\bar{\Omega}$. The crack is denoted by $\Gamma_{C}$ such that $\partial \Omega=\Gamma_{D} \cup \Gamma_{C}$. The initial triangulation is shown in Figure 5, and each triangulation is refined into a half-sized triangulation uniformly to get a higher level triangulation. Since the exact eigenvalues of this problem are unknown, we take the first eight eigenvalues by the conforming $\mathrm{P}_{3}$ element on the mesh $\mathcal{T}_{9}$ as reference 


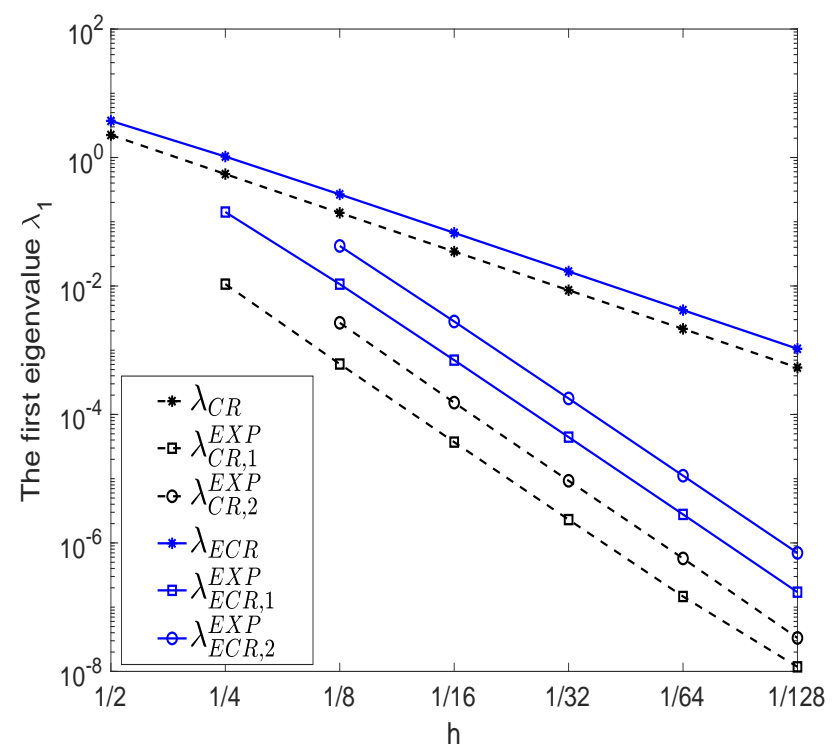

FIGURE 4. The errors of the extrapolation eigenvalues for Example 2.

eigenvalues. Since the convergence rates of eigenvalues are unknown, the extrapolation eigenvalues $\lambda_{\mathrm{CR}, 2}^{\mathrm{EXP}}$ in (3.58) and $\lambda_{\mathrm{ECR}, 2}^{\mathrm{EXP}}$ in (4.14) are considered for this example.
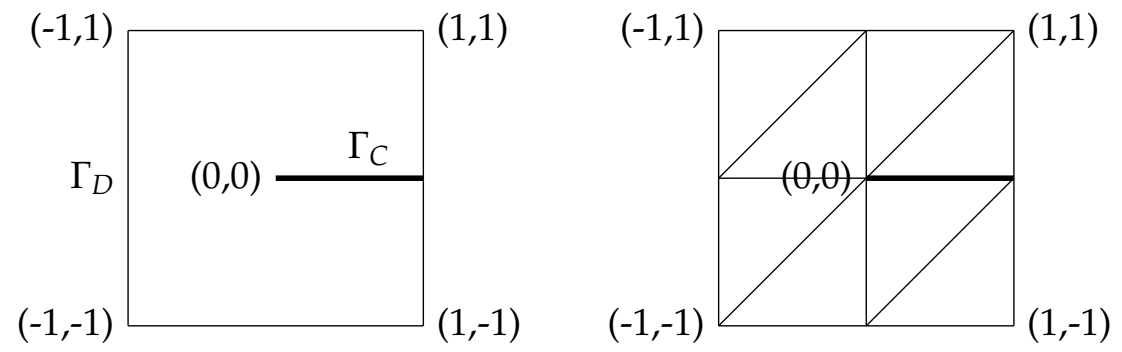

FIGURE 5. The crack domain $\Omega$ ( left) and the initial triangulation $\mathcal{T}_{1}$ ( right ) in Example 3.

For both the CR element and the ECR element, numerical experiments show that the first and the sixth eigenvalues converge at the rate 1 , and the other six eigenvalues converge at the optimal rate 2 . Figure 6 plots the errors of the eigenvalues $\lambda_{1}, \lambda_{2}, \lambda_{6}$ and $\lambda_{8}$ by both nonconforming elements. For $\lambda_{2}$ and $\lambda_{8}$, the corresponding eigenfunctions are smooth, and meets the regularity constraints of eigenfunctions in Theorem 3.2 and 4.1. As showed in Figure 6, the extrapolation eigenvalues $\lambda_{\mathrm{CR}, 2}^{\mathrm{EXP}}$ and $\lambda_{\mathrm{ECR}, 2}^{\mathrm{EXP}}$ improve the accuracy of the eigenvalues from $O\left(h^{2}\right)$ to $O\left(h^{4}\right)$, which confirms the theoretical results. For $\lambda_{1}$ and $\lambda_{6}$, the corresponding eigenfunctions are not smooth and the approximate eigenvalues by both nonconforming elements converge at the rate 1 . These two cases are not covered by the 
theory. For $\lambda_{1}$, the extrapolation eigenvalues $\lambda_{\mathrm{ECR}, 2}^{\mathrm{EXP}}$ admit a higher accuracy $O\left(h^{3}\right)$, and the eigenvalues $\lambda_{\mathrm{CR}, 2}^{\mathrm{EXP}}$ admit an even higher accuracy $O\left(h^{4}\right)$. Different from the case for $\lambda_{1}$, the convergence rate of the extrapolation eigenvalues $\lambda_{\mathrm{CR}, 2}^{\mathrm{EXP}}$ and $\lambda_{\mathrm{ECR}, 2}^{\mathrm{EXP}}$ for $\lambda_{6}$ is slightly larger than 1. Despite the small improvement in the convergence rates for these two cases, the accuracy of the eigenvalues is remarkably improved by the extrapolation methods.
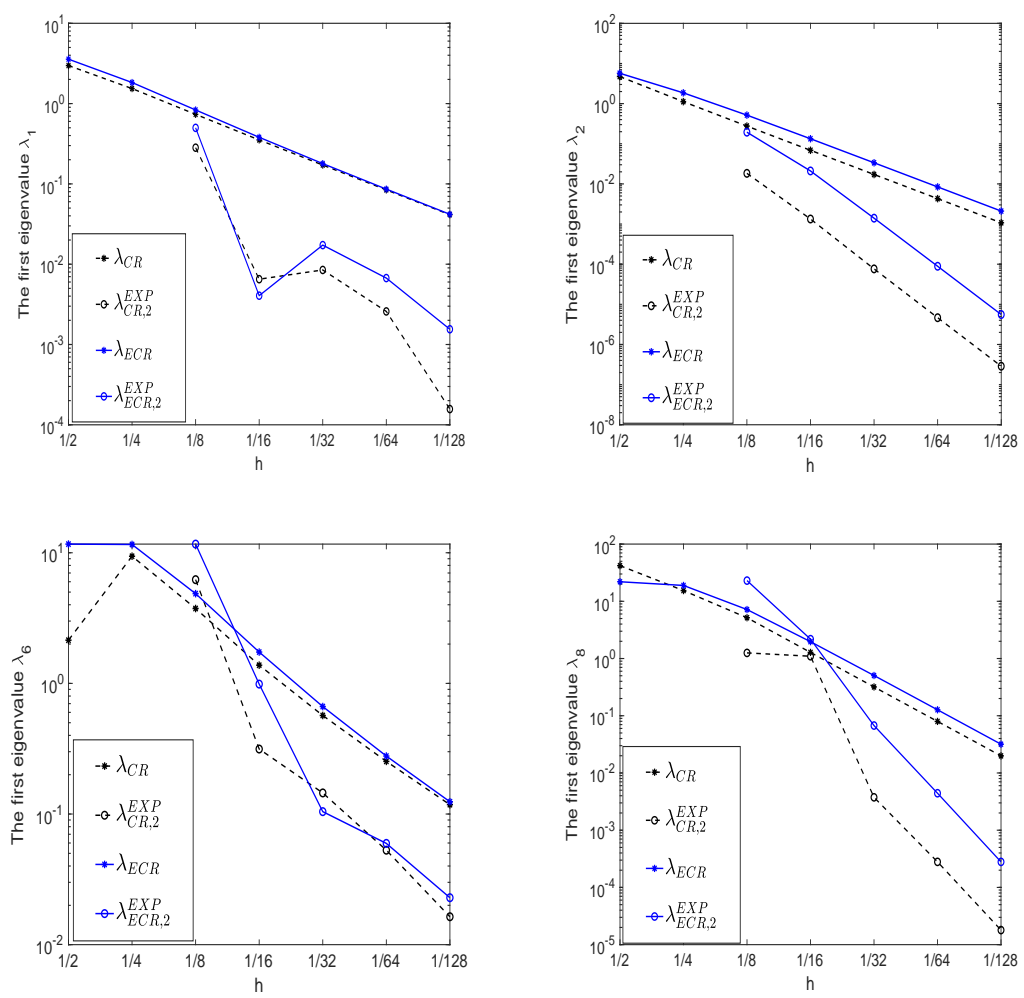

FiguRE 6. The errors of extrapolation eigenvalues for Example 3.

\section{REFERENCES}

[1] H Blum and R Rannacher. Finite element eigenvalue computation on domains with reentrant corners using Richardson extrapolation. Journal of Computational Mathematics, 8(4):321-332, 1990.

[2] Jan H Brandts. Superconvergence and a posteriori error estimation for triangular mixed finite elements. Numerische Mathematik, 68(3):311-324, 1994.

[3] Wei Chen and Qun Lin. Asymptotic expansion and extrapolation for the eigenvalue approximation of the biharmonic eigenvalue problem by Ciarlet-Raviart scheme. Advances in Computational Mathematics, 27(1):95-106, 2007. 
[4] Michel Crouzeix and Pierre-Arnaud Raviart. Conforming and nonconforming finite element methods for solving the stationary Stokes equations. Revue française d'automatique informatique recherche opérationnelle. Mathématique, 7(R3):33-75, 1973.

[5] Yanheng Ding and Qun Lin. Quadrature and extrapolation for the variable coefficient elliptic eigenvalue problem. Systems Science Mathematical Sciences, 3(4):327-336, 1990.

[6] Jim Douglas and Jean E Roberts. Global estimates for mixed methods for second order elliptic problems. Mathematics of Computation, 44(169):39-52, 1985.

[7] Ricardo Durán. Superconvergence for rectangular mixed finite elements. Numerische Mathematik, 58(1):287-298, 1990.

[8] Jun $\mathrm{Hu}$, Yunqing Huang, and Qun Lin. Lower bounds for eigenvalues of elliptic operators: By nonconforming finite element methods. Journal of Scientific Computing, 61(1):196-221, 2014.

[9] Jun Hu and Limin Ma. Asymptotically exact a posteriori error estimates of eigenvalues by the Crouzeix-Raviart element and enriched Crouzeix-Raviart element. SIAM Journal on Scientific Computing, 42(2):A797-A821, 2020.

[10] Jun Hu, Limin Ma, and Rui Ma. Optimal superconvergence analysis for the CrouzeixRaviart and the Morley elements. arXiv preprint arXiv:1808.09810v2, 2018.

[11] Jun Hu and Rui Ma. The Enriched Crouzeix-Raviart elements are equivalent to the Raviart-Thomas elements. Journal of Scientific Computing, 63(2):410-425, 2015.

[12] Yunqing Huang and Jinchao $\mathrm{Xu}$. Superconvergence of quadratic finite elements on mildly structured grids. Mathematics of computation, 77(263):1253-1268, 2008.

[13] Shanghui Jia, Hehu Xie, Xiaobo Yin, and Shaoqin Gao. Approximation and eigenvalue extrapolation of biharmonic eigenvalue problem by nonconforming finite element methods. Numerical Methods for Partial Differential Equations, 24(2):435-448, 2010.

[14] Yuwen Li. Global superconvergence of the lowest order mixed finite element on mildly structured meshes. SIAM Journal on Numerical Analysis, 56(2):792-815, 2018.

[15] Qun Lin. Can we compute laplace eigenvalues well, like computing $\pi$ ? International Journal of Information and Systems Sciences, 1(2):172-183, 2005.

[16] Qun Lin, Hung-Tsai Huang, and Zi-Cai Li. New expansions of numerical eigenvalues for $-\Delta u=\lambda \rho u$ by nonconforming elements. Mathematics of Computation, 77(264):20612084, 2008.

[17] Qun Lin, Hung-Tsai Huang, and Zi-Cai Li. New expansions of numerical eigenvalues by Wilson's element. Journal of Computational and Applied Mathematics, 225(1):213-226, 2009.

[18] Qun Lin and Jiafu Lin. Finite element methods: Accuracy and Improvement. China Sci. Press, Beijing, 2006.

[19] Qun Lin and Tao Lu. Asymptotic expansions for finite element eigenvalues and finite element. Bonn. Math. Schrift, 158:1-10, 1984.

[20] Qun Lin and Dongsheng Wu. High-accuracy approximations for eigenvalue problems by the carey non-conforming finite element. International Journal for Numerical Methods in Biomedical Engineering, 15(1):19-31, 1999.

[21] Qun Lin and Hehu Xie. Asymptotic error expansion and Richardson extrapolation of eigenvalue approximations for second order elliptic problems by the mixed finite 
element method. Applied Numerical Mathematics, 59(8):1884-1893, 2009.

[22] Qun Lin and Hehu Xie. New expansions of numerical eigenvalue for $-\Delta u=\lambda \rho u$ by linear elements on different triangular meshes. Internat Journal of Information Systems Sciences, 6:10-34, 2010.

[23] Qun Lin, Junming Zhou, and Hongtao Chen. Extrapolation of three-dimensional eigenvalue finite element approximation. Mathematics in Practice Theory, 11(11):132139, 2011.

[24] Ping Luo and Qun Lin. High accuracy analysis of the Adini's nonconforming element. Computing, 68(1):65-79, 2002.

[25] Luisa Donatella Marini. An inexpensive method for the evaluation of the solution of the lowest order Raviart-Thomas mixed method. SIAM Journal on Numerical Analysis, 22(3):493-496, 1985.

[26] Wang Ming and Jinchao Xu. The Morley element for fourth order elliptic equations in any dimensions. Numerische Mathematik, 103(1):155-169, 2006.

[27] Leslie Sydney Dennis Morley. The triangular equilibrium element in the solution of plate bending problems. The Aeronautical Quarterly, 19(2):149-169, 1968.

[28] Rolf Rannacher. Nonconforming finite element methods for eigenvalue problems in linear plate theory. Numerische Mathematik, 33(1):23-42, 1979.

[29] Pierre-Arnaud Raviart and Jean-Marie Thomas. A mixed finite element method for second order elliptic problems. Springer Berlin Heidelberg, (606):292-315, 1977.

LMAM and School of Mathematical Sciences, Peking University, Beijing 100871, P. R. China. huJUN@MATH.PKU.EDU.CN

LMaM and School of Mathematical Sciences, Peking University, Beijing 100871, P. R. China. malimINPKU@GMAIL.COM 